\title{
Modelo multifactor para analizar la exposición de los hedge funds a factores de riesgo macroeconómico*
}

\author{
(Multifactor Model for the Analysis of the Vulnerability \\ of Hedge Funds to Macroeconomic Risk Factors) \\ Elitania Leyva Rayón**
}

\section{RESUMEN}

Los hedge funds son fondos de inversión alternativa para grandes patrimonios e inversores institucionales. Dado que coexisten con los fondos tradicionales y otras entidades financieras en muchos de los mismos mercados y con los mismos activos, los hedge funds deberían responder a las mismas fuerzas macroeconómicas que influyen sobre aquéllos. Por ello, el propósito de este trabajo es estimar un modelo multifactor macroeconómico que pondere la importancia y la dirección que tiene la influencia de los factores de riesgo macroeconómico sobre estos fondos. Con ese fin, se utiliza una base de datos construida manualmente con información de la revista MARHedge Performance \& Evaluation Directory (1999-2006). Los resultados apoyan la hipótesis de que las sorpresas macroeconómicas que tienen poder explicativo sobre la rentabilidad de los activos estándar también lo tienen en el caso de los hedge funds.

Palabras clave: modelo multifactor, índices de hedge funds, sorpresas macroeconómicas.

Clasificación JEL: C22, C51, E44, G23.

\begin{abstract}
Hedge funds are alternative investment funds aimed at high fortunes and institutional investors. Since investing in many markets and assets in which traditional funds and other financial institutions do, hedge funds should respond to the same macroeconomic forces. Therefore, the purpose of this paper is to estimate a multifactor macroeconomic model in order to find the importance and direction of the influence of macroeconomic risk factors on hedge funds. We use a manually constructed database with information from the magazine MARHedge Performance \& Evaluation Directory (1999-2006). The analysis results support the hypothesis that the macroeconomic innovations (surprises) that have explanatory power on standard assets returns also have explanatory power on the returns of hedge funds.
\end{abstract}

Keywords: multifactor model, hedge funds indexes, macroeconomic innovations. JEL classification: C22, C51, E44, G23.

\footnotetext{
* Fecha de recepción: 31/07/2012. Fecha de aprobación: 17/09/2014.

** Profesora de tiempo completo del Departamento de Economía de la Escuela de Negocios y Economía, Universidad de las Américas Puebla. Tel.: (222) 22924 37. Correo electrónico: elitania. leyva@udlap.mx.
} 


\section{INTRODUCCIÓN}

Los hedge funds son fondos de inversión alternativa dirigidos a grandes patrimonios e inversores institucionales. Poseen una escasa regulación que les permite asumir estrategias y niveles de apalancamiento no alcanzables por otro tipo de fondos. Se estructuran como una sociedad de capital en la que el principal partícipe se convierte en su gestor, siendo el mayor responsable de las decisiones de inversión mientras que el resto de los socios son considerados inversores. Además de la comisión fija, los inversores pagan una comisión variable basada en el desempeño del gestor, quien usualmente invierte una parte significativa de su fortuna dentro del fondo con el fin de asegurar la alineación de los intereses económicos entre los socios. ${ }^{1}$

El punto de partida de esta investigación es que los hedge funds invierten en muchos de los mismos mercados y los mismos tipos de activos que los fondos tradicionales y otras entidades financieras y, por tanto, deberían responder a la influencia de las mismas fuerzas macroeconómicas. Entonces, el propósito de este trabajo es estimar un modelo multifactor macroeconómico con el objetivo de ponderar la importancia y la dirección de la influencia que ejercen los factores de riesgo macroeconómico sobre la rentabilidad de los hedge funds.

Para el desarrollo del modelo se crean índices de rentabilidad con la técnica de análisis factorial y se estima en series de tiempo mediante mínimos cuadrados ordinarios (MCO) y la matriz de White para el cálculo de errores estándar robustos. Además, se realizan los contrastes de especificación y diagnóstico necesarios para la comprobación de la calidad del modelo. Para su estimación fue utilizada una base de datos construida manualmente con la información de la revista MARHedge Performance \& Evaluation Directory (1999-2006). Los resultados del análisis empírico apoyan la hipótesis de que las que las sorpresas (componentes no esperados) en los factores de riesgo macroeconómico que tienen poder explicativo sobre la rentabilidad de los activos estándar también lo tienen sobre la rentabilidad de los hedge funds.

El trabajo se desarrolla de la siguiente manera: en la sección I se revisa la literatura relativa a los modelos multifactores macroeconómicos. Luego, en la II, se describe la base de datos construida manualmente y las metodologías a emplear. En la sección III se detalla la construcción de las variables depen-

${ }^{1}$ Para una descripción más detallada de las características de este tipo de negocios, se puede consultar Caslin (2004). 
dientes e independientes; la exposición del modelo multifactor y los resultados del análisis empírico se realiza en la sección IV. Finalmente, se presentan las conclusiones.

\section{REVISIÓN DE LA LITERATURA}

La relevancia de los modelos multifactores macroeconómicos desde la perspectiva de la arbitrage princing theory (teoría del arbitraje de precios o APT, por sus siglas en inglés) quedó asentada a partir del influyente trabajo de Chan, Chen y Hsieh (1985), quienes analizaron el efecto tamaño mediante los precios de cotización de las empresas. Estos autores elaboraron veinte carteras con las empresas que cotizaban en el índice New York Stock Exchange (NYSE) entre 1958 y 1977, cada una con el mismo número de activos. Al utilizar el logaritmo natural del valor de mercado de la empresa como variable para medir indirectamente el tamaño, encontraron que las empresas pequeñas poseen coeficientes beta $(\beta)$ de mercado más altos que las grandes empresas respecto a los factores de riesgo macroeconómico empleados (tasa de crecimiento mensual de la producción industrial, inflación esperada y no esperada, tipo de interés real esperado, la term structure, el default premium y el índice NYSE). ${ }^{2}$ Por otra parte, utilizaron un factor de riesgo basado en la tasa de crecimiento de la formación neta de negocios que varía directamente con las expansiones y contracciones económicas. Encontraron que las empresas pequeñas tienen rentabilidades esperadas más altas debido a sus elevadas covariaciones con los cambios en las condiciones de los negocios.

Otro trabajo clave es el de Chen, Roll y Ross (1986), que explora un conjunto de variables macroeconómicas que influyen sobre los precios de los activos y, por tanto, sobre su rentabilidad. Dado que su objetivo es modelar las rentabilidades de los activos como funciones de variables macroeconómicas, los mercados de activos son vistos como endógenos en relación con otros mercados, mientras que las variables macroeconómicas son consideradas como fuerzas exógenas. ${ }^{3}$ Estos investigadores utilizaron observaciones mensuales de un con-

\footnotetext{
${ }^{2}$ Las definiciones de las sorpresas term structure y default premium pueden consultarse en el cuadro 2.

${ }^{3}$ Señalan que cualquier variable que sea necesaria para completar la descripción del estado de naturaleza deberá ser parte de los factores de riesgo. Por ejemplo, el desempleo, que aunque no tiene una influencia directa sobre los flujos de fondos sí puede describir los cambios en las oportunidades de inversión.
} 
junto de variables de estado, tales como la tasa de crecimiento anual y mensual de la producción industrial, la inflación esperada y la no esperada, el tipo de interés real, el default premium y la term structure. Entre sus principales resultados, observaron una correlación relativamente alta entre las series de producción industrial anual y mensual, así como entre las variables default premium y term structure.

Li (2002) trata de explicar la exposición común de la rentabilidad de activos y de bonos del gobierno a factores de riesgo macroeconómico (tipo de interés real, tipo de interés no esperado, inflación esperada y no esperada, term structure y default premium). El vínculo entre la correlación de las rentabilidades de activos y los bonos con los factores macroeconómicos es examinado con tres formulaciones sobre la dinámica de la rentabilidad. La primera se refiere a un modelo de regresión lineal para vincular la correlación no condicional entre las rentabilidades con la incertidumbre de los factores macroeconómicos. La segunda formulación modela las autocorrelaciones en la media y la volatilidad de la rentabilidad de los activos. La tercera identifica las autocorrelaciones de las rentabilidades de los activos, de los bonos y de ambos, mediante un modelo de vector de autorregresión. Sus resultados indican que las principales tendencias en la correlación entre activos y bonos están determinadas principalmente por la incertidumbre a largo plazo de la inflación esperada, mientras que la inflación no esperada y el tipo de interés real son significativos sólo en menor grado.

Clare y Thomas (1994), presentan evidencia empírica de la influencia de factores de riesgo macroeconómico sobre la rentabilidad de los activos del mercado financiero del Reino Unido para el periodo de enero de 1983 a diciembre de 1990. Utilizan 18 factores macroeconómicos, de los cuales algunos están a nivel, mientras que otros, en primeras diferencias y en primeras diferencias logarítmicas. Sin embargo, a diferencia de Chan, Chen y Hsieh (1985), y Chen, Roll y Ross (1986), consideran que la inclusión de un índice de mercado es inapropiada en la identificación de factores macroeconómicos que influyen en el precio de los activos. ${ }^{4}$ Encuentran que las repercusiones en la inflación están asociadas a un signo positivo en los activos, lo que sugiere que éstos no son considerados como cobertura frente a la inflación en el periodo analizado.

\footnotetext{
${ }^{4}$ Además, Chan, Chen y Hsieh (1985), y Chen, Roll y Ross (1986) no observan un rol significativo del índice de mercado que emplean cuando es añadido al conjunto de variables macroeconómicas.
} 
Por su parte, Antoniou, Garrett y Priestley (1998) estudian el comportamiento de los activos del London Stock Exchange suponiendo la presencia de factores generalizados comunes. ${ }^{5} \mathrm{~A}$ partir de dos muestras estiman las sensibilidades entre la rentabilidad y los factores de riesgo macroeconómico, y las primas de riesgo que validan dicha relación. Los factores utilizados son: inflación no esperada, cambios en la inflación esperada, term structure, default premium, índice de mercado y repercusiones no esperadas (en la producción industrial, en las ventas al menudeo, en la oferta monetaria, en los precios de las materias primas y en el tipo de cambio). Estas repercusiones son generadas a partir del componente no observado o mediante modelos autorregresivos con parámetros de variables de tiempo, que permiten a los agentes actualizar sus expectativas recursivamente cada periodo con base en la información disponible en el momento que las forman. Mediante regresiones en sección cruzada, encuentran que el modelo es capaz de explicar una cantidad sustancial de la variación de las rentabilidades medias de los activos para ambas muestras y que éstas parecen ajustarse a una estructura de factor aproximado.

Erdinç (2003) investiga los efectos de los factores de riesgo macroeconómico sobre la rentabilidad de los activos de las economías alemana y turca a través de un modelo multifactor para cada país. Señala que la economía de Alemania es propia de un país industrializado y con un mercado accionario relativamente maduro, mientras que la economía de Turquía posee un joven mercado accionario emergente. Para cada una (y subperiodos en el caso alemán) construye veinte carteras en orden alfabético con aproximadamente el mismo número de activos. A través de un análisis factorial (por componentes principales y por máxima verosimilitud) extrae los más importantes factores de ocho variables macroeconómicas más un índice de mercado para cada país, y mediante MCO halla que la significación de todos los coeficientes beta aumenta cuando se agrega el índice de mercado en cada economía. Señala que, a diferencia del modelo de la economía alemana, ${ }^{6}$ el de la economía turca no es capaz de obtener una variable macroeconómica con influencia, lo que podría deberse a las condiciones estructurales de este mercado, joven, con un reducido número de empresas y un volumen de activos negociados relativamente bajo.

\footnotetext{
${ }^{5}$ Seleccionan al azar 138 activos y los dividen en dos muestras, para el periodo que va de enero de 1980 a agosto de 1993 .

${ }^{6}$ En el caso de esta economía, encuentra evidencia de que la prima de riesgo del tipo de interés no esperado y de la inflación no esperada tiene efectos significativos sobre su mercado accionario.
} 
Azeez y Yonezawa (2006) examinan la rentabilidad del mercado de Japón durante el episodio de su burbuja económica en los años ochenta. Los factores macroeconómicos que utilizan son los cambios no esperados en la oferta monetaria, la inflación, la term structure, el tipo de cambio, la tasa de producción industrial y el índice comercial de precios de la tierra. Este último es seleccionado dadas las características particulares de ese país. No obstante, en los resultados del modelo se observa que este factor no tiene efecto sobre el precio de los activos. De igual manera, el tipo de interés libre de riesgo que utilizan no es el del Treasury-bill a un mes, dado que en Japón no se utiliza este tipo de interés. Señalan que al utilizar sorpresas macroeconómicas no es raro encontrar alta correlación entre la inflación y la term structure debido a que estos dos factores están relacionados con el tipo de interés nominal. Emplean regresiones aparentemente no relacionadas y observan que los signos de estas variables son estables (en términos del valor de los coeficientes y de los signos negativos) entre los periodos analizados y que la magnitud de las primas de riesgo se incrementó durante y después de la burbuja.

Bailey y Chan (1993) estudian la rentabilidad de los mercados de materias primas y su relación con los mercados de activos y de bonos, con el fin de encontrar una variación común entre estos tres tipos de mercado a partir de su exposición a factores de riesgo macroeconómico. Para ello, emplean datos de 21 materias primas diferentes durante el periodo 1966-1987 y observan que cuando se espera que la actividad real sea alta, las variables de estado tienden a tener un valor bajo. Sus resultados sugieren que las primas de riesgo en los mercados de activos y de bonos están correlacionadas con fuerzas similares a las que afectan a los mercados de materias primas, es decir, las primas de riesgo ganadas por la exposición a factores de riesgo son las mismas en los mercados de activos, de bonos y de materias primas. También encuentran que el spread (margen de compraventa) entre el precio de las materias primas al contado y los precios futuros está afectado por los riesgos macroeconómicos comunes a todos los mercados. Además, los precios futuros de las materias primas están correlacionados con el índice de rentabilidad del mercado de activos y con el spread de los bonos de las empresas de baja y alta calificación. Señalan que alrededor de $40 \%$ de la variación de una cartera compuesta por rentabilidades de materias primas con sensibilidad a la actividad económica está explicada por la rentabilidad de activos y de bonos.

Chen y Jordan (1993) investigan la capacidad de dos modelos multifactores para predecir la rentabilidad mensual de carteras creadas con empresas que 
cotizaban en los índices NYSE y AMEX. La diferencia entre los modelos estriba en el número y naturaleza de los factores empleados. En el estadístico, los factores son extraídos mediante análisis factorial, mientras que en el macroeconómico los factores son sorpresas en variables macroeconómicas.

Sin embargo, las variaciones observadas en los resultados de ambos modelos son relativamente pequeñas: las $R^{2}$ en el modelo estadístico son más altas que en el macroeconómico (41 de 69), pero las diferencias son muy pequeñas; de hecho el promedio de las $69 R^{2}$ es el mismo en los dos modelos (53\%). Mediante las pruebas de Davison-Mackinnon, $U^{2}$ de Theil y de Wilcoxon, concluyen que el modelo estadístico tiene un poder de predicción de rentabilidades sólo marginalmente superior, lo que no es de extrañar dado que los cinco factores fueron extraídos de las mismas rentabilidades que se tratan de explicar, de manera que los resultados en la muestra original no son resultados generales. Por ello, aplicaron las mismas metodologías a una muestra de datos distinta (treinta carteras), con resultados que favorecen al modelo macroeconómico (excepto en la prueba de Wilcoxon).

Por otro lado, Connor (1995) emplea 108 rentabilidades mensuales de 779 activos estadounidenses de alta capitalización durante el periodo de enero de 1985 a diciembre de 1993, para analizar el poder explicativo de tres tipos de modelos multifactoriales (estadístico, fundamental y macroeconómico). Observa que, en términos de capacidad de explicación, los modelos fundamentales y estadísticos son superiores a los macroeconómicos, y que a su vez los primeros superan ligeramente a los segundos.

También encuentra que los factores macroeconómicos agregan un poder explicativo marginal cuando son combinados con factores fundamentales, lo que puede sugerir que éstos (en alguna combinación desconocida) podrían estar adquiriendo las mismas características de riesgo que los factores macroeconómicos. Menciona que una posible explicación de estos resultados sería la existencia de un gran número de fuentes de datos externos usados en los modelos fundamentales, particularmente en el enorme conjunto de variables dummy de industrias.

Finalmente, Das, Kish y Muething (2005) modelan rentabilidades de hedge funds mediante la metodología usada por Chen, Roll y Ross (1986) y los cinco factores macroeconómicos empleados en Connor (1995) con el objetivo de que los inversores puedan darse una idea mejor del tipo y la cantidad de riesgo que corren al invertir en este tipo de fondos. Utilizan la rentabilidad mensual de los hedge funds de la base de datos $\mathrm{ZCM} /$ Hedge, que clasifica los fondos 
en cuatro clases y diez estrategias de inversión. ${ }^{7}$ Analizan la rentabilidad de los hedge funds poniéndola a prueba con factores de riesgo macroeconómico que afectan a los fondos tradicionales. Las regresiones en series de tiempo son realizadas por cada estrategia seguida (en lugar de individualmente) a fin de reducir los errores en las variables (errores en la correcta medición de las betas), como la posibilidad de autocorrelación. El modelo que incluye los cinco factores explica aproximadamente $25 \%$ de la variación de la rentabilidad de los hedge funds y la significación de los coeficientes no varía mucho en los diferentes periodos de prueba. Observan que los coeficientes para las variables default premium y term structure son estadísticamente significativos para todas las categorías cuando se analiza el periodo completo, por tanto, concluyen que estas dos variables son las que tienen mayor poder explicativo de la rentabilidad de los hedge funds en general.

\section{BASE DE DATOS Y METODOLOGÍAS}

Recopilar datos sobre hedge funds es uno de los mayores problemas a los que se enfrentan los investigadores, ya que no están regulados por organismos oficiales y, por tanto, no tienen la obligación de hacer pública su información. Esta falta de transparencia en su información interna genera varias limitaciones para su estudio. Por fortuna, existen hedge funds que proporcionan información de manera voluntaria a las principales bases de datos. ${ }^{8}$ No obstante, es limitada, ya que envían sólo la que consideran necesaria para atraer nuevos inversores.

Para realizar el análisis empírico en este estudio, se ha construido manualmente una base de datos ${ }^{9}$ a partir de la información sobre hedge funds publicada en la revista MARHedge Performance \& Evaluation Directory (1999-2006), perteneciente a la base de datos MARHedge, para el periodo $1999-2006 .{ }^{10} \mathrm{La}$ in-

\footnotetext{
${ }^{7}$ Las clases son: on-shore y off-shore; y fondos de fondos: on-shore y off-shore. Utilizan tres subperiodos de 48 meses cada uno: de enero a diciembre de 1989-1992, 1993-1996, y 1997-2000; y el periodo completo.

${ }^{8}$ Las principales bases de datos privadas sobre hedge funds son: Tremont Advisory Shareholders Services (TASS), Manager Account Reports (MARHedge) y Hedge Fund Research, Inc. (HFRI).

${ }^{9}$ De la misma manera, Brown, Goetzmann e Ibbotson (1999) recolectaron manualmente la información de The US. Offshore Directory y a partir de esta guía crearon una base con información anual.

${ }^{10}$ En 1994 fue lanzada la base de datos MAR, durante la primera Annual International Conference on Hedge Fund Investments, en las Bermudas. En marzo de 2002, la base de datos y la propiedad intelectual de MAR fue comprada por Zurich Capital Markets Inc. Finalmente, en 2006
} 
formación de los fondos activos se extrajo de la última edición con información cuantitativa (segundo trimestre de 2006), mientras que la de los fondos liquidados se obtuvo mediante la construcción de un cementerio, ya que para el periodo analizado dicha publicación no cuenta con una sección sobre ellos.

El cementerio se elaboró de la siguiente manera: se comparó el primer número de MARHedge Performance \& Evaluation Directory (enero-junio de 1999) del periodo considerado con el siguiente (julio-diciembre de 1999) y los hedge funds que aparecen en el primero, pero no en el segundo fueron catalogados como liquidados y agrupados de acuerdo con la estrategia que siguieron; después, se repitió el procedimiento con este segundo número del periodo y el tercero (enero-junio de 2000), y así sucesivamente hasta abarcar todos los números de la revista del lapso 1999-2006 con información cuantitativa, habiendo obtenido un total de 1761 hedge funds liquidados ${ }^{11}$ y 3097 activos, repartidos en nueve estrategias (event driven, global emerging, global established, global international, global macro, long only/leverage, market neutral, sector, short-sellers). ${ }^{12}$

Siguiendo a Chen y Jordan (1993), ${ }^{13}$ se realizó un análisis factorial de la rentabilidad de los hedge funds de la base de datos construida para extraer un

fue vendida a Euromoney Institutional Investor, cambiando su nombre a MARHedge Institutional Investor. Desgraciadamente, a partir del segundo semestre de 2006, MARHedge Performance \& Evaluation Directory dejó de publicar la valiosa información cuantitativa interna de los fondos y llenó sus páginas con textos relativos a relaciones públicas, congresos, eventos, cenas de gala y diversas cuestiones sociales. Por ello, la información utilizada en este trabajo corresponde al lapso en que se publicó información interna cuantitativa con la que se pueden realizar análisis empíricos. Ésta es una de las principales limitaciones de esta investigación, ya que sería muy enriquecedor ampliar el periodo con el fin de contrastar los resultados de antes y después de la crisis financiera de 2007, es decir, analizar si los factores macroeconómicos cambiaron su influencia y significación respecto a los índices de hedge funds (activos y liquidados) por estrategia seguida después de la crisis o se mantuvieron constantes las relaciones previas a la crisis. La revista puede consultarse en la Universidad de Harvard, la Universidad de Pensilvania y en el MIT.

${ }^{11}$ Aunque la desaparición de un hedge fund puede deberse a motivos distintos a la liquidación (fusiones, cierre a nuevos inversores, etc.), Liang (2001) señala que la razón principal por la que un fondo es excluido de una base es el pobre desempeño en su rentabilidad, lo que puede interpretarse como quiebra.

${ }^{12}$ En los resultados del análisis empírico se describe cada una de estas estrategias (sección IV.1.b).

${ }^{13}$ Estos autores analizan las rentabilidades mensuales de carteras formadas por empresas con el mismo código de la Standard Industrial Classification (SIC) a partir de dos modelos: uno estadístico y uno macroeconómico. Realizan un regresión de las cargas de los cinco factores extraídos de las sorpresas macroeconómicas para encontrar las relaciones existentes. Destacan que, a diferencia del modelo estadístico, el macroeconómico tiene la atractiva característica de la interpretación económica de sus factores. 
determinado número de factores que empleamos como índices de rentabilidad (variables dependientes). La aplicación de esta técnica tiene por objetivo condensar la información contenida en las rentabilidades mensuales de los hedge funds de cada una de las estrategias seguidas -dentro del grupo de activos y dentro del grupo de liquidados- en un conjunto de factores más pequeño. ${ }^{14} \mathrm{Se} \mathrm{hizo}$ un análisis factorial de cada una de las estrategias dentro del grupo de hedge funds activos y otro aparte de las estrategias seguidas en el grupo de fondos liquidados, debido a que si se combinaran activos, liquidados y las nueve estrategias, las correlaciones resultantes y la estructura de factores sería una representación pobre de la estructura única de cada estrategia seguida dentro de cada grupo.

Por otro lado, las sorpresas en los factores de riesgo macroeconómico que se utilizan como variables independientes se obtienen a partir de un modelo autorregresivo, de primeras diferencias y de cálculos aritméticos. ${ }^{15}$ Después de construir las variables, se probó el modelo multifactor en series temporales mediante MCO y la matriz de White. No obstante, con la finalidad de comprobar la solidez del modelo, es conveniente especificar correctamente las relaciones a estimar y someterlo a diversas pruebas estadísticas a partir de la formulación de un conjunto de hipótesis que permitirán confirmar su validez y calidad.

Existen diferentes pruebas de especificación y diagnóstico relativas a la parte sistemática del modelo y a las propiedades que debe cumplir la perturbación aleatoria. Los diversos errores que en ocasiones se generan en el proceso se corrigen reespecificando el modelo e incorporando las correcciones oportunas. Las pruebas de diagnóstico se realizaron mediante el estudio de los residuos del modelo, pues éstos dejan de cumplir las hipótesis planteadas cuando se producen errores de especificación. De manera que el modelo fue sometido a las pruebas de estacionariedad (prueba de Dickey-Fuller aumentada), multicolinealidad (matriz de correlaciones), autocorrelación (prueba de

\footnotetext{
${ }^{14}$ En esta línea, López-Pascual y Camarero (2013) señalan que a través de un análisis explícito (cuyo objetivo es identificar y medir la sensibilidad de los factores reales que explican las rentabilidades históricas, por ejemplo, modelar la rentabilidad como una función lineal de varios factores macroeconómicos o índices) o un análisis implícito (cuyo objetivo es identificar ciertos factores estadísticos que explican las rentabilidades históricas, por ejemplo, el análisis de componentes principales, que ocupa factores explicativos que poseen la mayor varianza posible con la restricción de que cada factor debe ser ortogonal a los componentes anteriores) se puede tratar de explicar la rentabilidad de los hedge funds y la clasificación de las estrategias de inversión.

${ }^{15}$ La construcción detallada de las sorpresas macroeconómicas puede ser consultada en el cuadro 2 .
} 
Breusch-Godfrey), heterocedasticidad (prueba de White), normalidad (prueba de Jarque-Bera) y linealidad (prueba de Ramsey). Una vez realizados los contrastes se llevaron a cabo los ajustes necesarios mediante un modelo $\operatorname{AR}(p)$, un $\operatorname{MA}(q)$ o un $\operatorname{ARMA}(p, q) .{ }^{16}$

\section{CONSTRUCCIÓN DE LAS VARIABLES}

\section{Variables dependientes: índices de rentabilidad}

Con el propósito de conocer el número mínimo de dimensiones capaces de explicar el máximo de información contenida en las rentabilidades mensuales de los hedge funds, cada una de las estrategias seguidas por dichos fondos fue sometida a un análisis factorial, tanto los activos como los liquidados. ${ }^{17}$ A partir de los factores obtenidos se calculó la puntuación factorial de cada observación mensual mediante el método de regresión, lo que se utilzó como índices de rentabilidad.

Para ello, se utilizaron los 1761 hedge funds liquidados y los 3097 activos de la base de datos construida. De estos últimos, existe información sobre la rentabilidad de 2 645, pero sólo en 994 casos esta información es mensual para el periodo de enero de 2002 a junio de 2006. En tanto, de los 1761 liquidados, se dispone de información sobre la rentabilidad 1016, pero sólo la de 383 es mensual, y de éstos únicamente en 190 casos corresponde al periodo de enero de 2002 a septiembre de $2005 .{ }^{18}$ Por tanto, al seleccionar fondos con información sobre sus rentabilidades mensuales para el periodo más largo posible, la muestra disminuyó hasta un total de 190 liquidados y 994 activos. La restricción relativa al periodo de análisis se impuso con el objetivo de abarcar el mayor lapso posible sin que falte ninguna observación.

Para el análisis empírico se contó con 54 rentabilidades mensuales para cada uno de los hedge funds activos, mientras que para cada uno de los liquida-

\footnotetext{
${ }^{16}$ Las primeras cuatro pruebas son básicas y deben pasarse con éxito en el $100 \%$ de los casos debido a su relevancia. Sin embargo, no es imprescindible el cumplimiento de las dos últimas, ya que se trata de pruebas complementarias que tienen una importancia menor cuando se trata de MCO.

${ }^{17}$ Los análisis factoriales se realizaron con el paquete de cómputo estadístico SPSS 15.0. Por simplicidad en la exposición, sólo se presentan los resultados. Las tablas correspondientes pueden solicitarse a la autora para su consulta.

${ }^{18}$ Una vez que el hedge fund deja de enviar su información, las bases de datos lo eliminan, lo que da como resultado que exista aún menos información sobre los fondos liquidados.
} 
dos fueron $45 .{ }^{19}$ Sin embargo, dado que el análisis factorial requiere que el número de observaciones (rentabilidades mensuales) sea superior al número de variables (hedge funds), fueron creados grupos con menos de 54 fondos para cada estrategia de los activos y menores a 45 fondos para cada estrategia de los liquidados. ${ }^{20}$ Además, a partir de la medida de tamaño muestral KMO (de Kaiser, Meyer y Olkin) y de la medida de tamaño individual MSA (measure of sampling adecuacy), se descartaron los fondos con un valor de la MSA inferior a 0.50 y que condujeran a una reducción en la medida KMO, ya que aportaban muy poca información al análisis.

Por otra parte, para evitar un doble peso de fondos con rentabilidad esencialmente idéntica, se han omitido los que tienen un mismo gestor, ya que las correlaciones entre ellos eran superiores a 0.95 , lo que no es de extrañar, ya que las políticas de inversión de un gestor son casi iguales en todos los fondos a su cargo. Además, el no omitir fondos esencialmente idénticos dificulta el análisis factorial debido a la información redundante que se halla en el proceso de extracción de factores, por lo que se usaron fondos con rentabilidades diferentes al resto. ${ }^{21}$ De este modo, del número original fueron seleccionados aquellos hedge funds con KMO y MSA superiores a 0.50 por grupo en cada estrategia seguida y con rentabilidades distintas entre ellos. Así, finalmente se usaron 687 fondos activos y 109 liquidados en los análisis factoriales. Estos se aplicaron a 33 grupos (25 de fondos activos y 8 de liquidados) por estrategia seguida. Cabe mencionar que para el conjunto de fondos liquidados no fue necesario crear más de un grupo por estrategia, ya que para todas ellas se dispone de menos de 45 fondos. ${ }^{22}$

\section{a) Resultados del análisis factorial}

Se comienza con la revisión de los principales estadísticos que nos ayudan a conocer la calidad de los análisis factoriales. Al respecto, las matrices de coeficien-

${ }^{19}$ De acuerdo con Brown, Goetzmann e Ibbotson (1999), la edad promedio de los fondos difícilmente llega a superar los tres años de actividad, debido principalmente a su pobre rentabilidad.

${ }^{20}$ Desafortunadamente, a diferencia de los fondos activos, no se dispone de ningún fondo liquidado que haya seguido la estrategia global international por un periodo mínimo de 45 meses.

${ }^{21}$ Los criterios para la inclusión de los fondos, sucesivamente, fueron: a) el número de rentabilidades mensuales, b) los activos gestionados y c) el orden alfabético.

${ }^{22}$ Excepto la estrategia market neutral, para la cual se dispone de 88 fondos. Sin embargo, después del análisis factorial de los dos grupos de esta estrategia (de 44 fondos, cada uno), fueron descartados casi cincuenta fondos debido a que obtuvieron una MSA inferior a 0.50 . 
tes de correlación nos muestran que se cumple el principal supuesto del que parte el análisis, ya que existen altas correlaciones entre los hedge funds utilizados. En todos los grupos, la correlación media es superior a 0.30 , lo que revela que hay un número sustancial de correlaciones que justifica el análisis. Además, la mayor parte de los coeficientes no redundantes son estadísticamente significativos a $90 \%$ de confianza. Al observar las correlaciones mínimas, medias y máximas en valores absolutos- y su significación, encontramos que, por ejemplo, de los 406 coeficientes de correlación no redundantes de los fondos activos que siguen la estrategia global macro, $88 \%$ son estadísticamente significativos. En las matrices de coeficientes de correlación también obtenemos el estadístico determinante, que es un indicador del grado de correlación y que al tener un valor muy bajo confirma que las correlaciones entre los fondos son altas.

Por otra parte, la medida KMO está muy próxima a la unidad en todos los grupos de las estrategias, lo que indica una buena adecuación de los datos en cada modelo y, en consecuencia, un buen ajuste factorial para cada grupo por estrategia en el conjunto de fondos activos y en el conjunto de fondos liquidados. Por otro lado, la prueba de esfericidad de Bartlett confirma que todas las matrices de correlación son significativamente distintas a la matriz de identidad, lo que refuerza al análisis. Por su parte, los valores mínimos y máximos de la MSA indican que los fondos utilizados son adecuados para su tratamiento mediante el análisis factorial, ya que todos obtuvieron un valor superior a 0.50 .

A partir de las matrices de correlación reproducidas se comprueba que los modelos factoriales estimados son adecuados, ya que las diferencias o residuos entre los coeficientes de correlación observados y los coeficientes de correlación reproducidos de cada matriz son muy pequeñas. ${ }^{23}$ Por ejemplo, de los 351 residuos no redundantes de los fondos liquidados con estrategia global established, sólo 75 (21\%) tienen valores absolutos superiores a 0.05 lo cual es aceptable para la fiabilidad del análisis. Por otro lado, la existencia de factores comunes entre los fondos utilizados se comprueba en las matrices de correlación antiimagen, ya que en todas ellas los negativos de los coeficientes de correlación parcial entre cada par de fondos son muy pequeños. ${ }^{24}$

Después de examinar los aspectos descriptivos, se extrajeron los factores en cada uno de los grupos por estrategia de hedge funds activos y liquidados. Por

\footnotetext{
${ }^{23}$ Excepto el modelo de la estrategia global emerging de los fondos liquidados, debido a que $66 \%$ de los residuales no redundantes fueron superiores a 0.05 .

${ }^{24}$ El porcentaje de los coeficientes de correlación parcial no redundantes -en valor absolutofue inferior a 0.40 .
} 
otro lado, mediante el método de ejes principales,${ }^{25}$ fueron extraídas las comunalidades, las cuales fueron altas debido a que se utilizó un elevado número de fondos. Entre los resultados, se halla que uno de los fondos activos con estrategia global established es el mejor representado, con una comunalidad de 0.999 , es decir, su varianza es reproducida por los factores comunes en $99.9 \%$. Cabe destacar que en todos los grupos la varianza media fue superior a $50 \%$.

\section{Cuadro 1. Número de factores extraídos y varianza total explicada en cada grupo} por estrategia seguida en cada uno de los conjuntos de fondos activos y liquidados

\begin{tabular}{|c|c|c|}
\hline \multirow[b]{2}{*}{ Estrategias } & \multicolumn{2}{|c|}{ Fondos activos } \\
\hline & $\begin{array}{c}\text { Número } \\
\text { de factores } \\
\text { extraídos }\end{array}$ & $\begin{array}{c}\text { Varianza total } \\
\text { explicada por el } \\
\text { número de } \\
\text { factores extraídos }\end{array}$ \\
\hline Event driven 1 & 3 & 62.1 \\
\hline Event driven 2 & 3 & 61.8 \\
\hline Event driven 3 & 3 & 60.2 \\
\hline Global emerging 1 & 3 & 59.3 \\
\hline Global emerging 2 & 2 & 66.6 \\
\hline Global established 1 & 3 & 69.8 \\
\hline Global established 2 & 3 & 61.1 \\
\hline Global established 3 & 3 & 59.7 \\
\hline Global established 4 & 3 & 62.6 \\
\hline Global international & 2 & 66.6 \\
\hline Global macro & 3 & 62.8 \\
\hline Long only / leverage & 3 & 69.5 \\
\hline Market neutral 1 & 3 & 72.0 \\
\hline Market neutral2 & 3 & 62.2 \\
\hline Market neutral 3 & 4 & 81.4 \\
\hline Market neutral 4 & 3 & 55.6 \\
\hline Market neutral 5 & 2 & 56.8 \\
\hline Market neutral 6 & 4 & 60.2 \\
\hline Market neutral 7 & 2 & 55.8 \\
\hline Market neutral 8 & 2 & 59.5 \\
\hline Market neutral 9 & 4 & 67.9 \\
\hline Market neutral 10 & 3 & 57.9 \\
\hline Sector 1 & 3 & 56.5 \\
\hline Sector 2 & 3 & 61.5 \\
\hline Short-sellers & 2 & 73.3 \\
\hline
\end{tabular}

\begin{tabular}{|l|c|c|}
\hline \multirow{2}{*}{ Estrategias } & \multicolumn{2}{|c|}{ Fondos liquidados } \\
\cline { 2 - 3 } & $\begin{array}{c}\text { Número de } \\
\text { factores } \\
\text { extraídos }\end{array}$ & $\begin{array}{c}\text { Varianza total } \\
\text { explicada por el } \\
\text { número de } \\
\text { factores extraídos }\end{array}$ \\
\hline Event driven & 4 & 80.2 \\
\hline Global emerging & 1 & \\
\hline & & 63.9 \\
\hline Global established & 4 & 62.6 \\
\hline & & \\
\hline Short-sellers & & \\
\hline Llobal macro & 1 & \\
\hline Market neutral & 3 & \\
\hline & & \\
\hline & & \\
\hline & & \\
\hline & & \\
\hline & & \\
\hline & & \\
\hline & & \\
\hline & & \\
\hline & & \\
\hline & & \\
\hline & & \\
\hline & & \\
\hline & & \\
\hline & & \\
\hline & & \\
\hline
\end{tabular}

Fuente: Elaboración propia.

${ }^{25}$ Cuando se utilizan más de treinta variables, todos los métodos de extracción tienden a dar la misma solución. 
El cuadro 1 muestra la varianza total explicada por los factores extraídos de los fondos activos y liquidados en cada una de las estrategias. ${ }^{26}$ En él se puede apreciar que, por ejemplo, los dos factores extraídos de los fondos liquidados que siguieron la estrategia short-sellers explican $97.4 \%$ de la varianza total. Conocido el número de factores extraídos para cada grupo por estrategia dentro del conjunto de fondos activos y dentro del conjunto de liquidados, se utilizaron las matrices de factores obtenidas para interpretar los factores. ${ }^{27}$ Como era de esperarse en el primer factor de cada grupo de estrategias en el conjunto de activos y en el conjunto de liquidados aparece un gran número de fondos con elevadas cargas factoriales. Después de extraer los factores calculamos las puntuaciones factoriales mediante el método de regresión y obtenemos así los índices de rentabilidad utilizados como variables dependientes.

\section{Variables independientes: sorpresas macroeconómicas}

Las sorpresas de los factores de riesgo macroeconómico que se utilizan como variables independientes en el modelo multifactor se describen en el cuadro 2 . A partir de la construcción de las variables dependientes e independientes ${ }^{28}$ se estimó el modelo multifactor. En la siguiente sección se presenta dicho modelo y los resultados.

\section{ESTIMACIÓN DEL MODELO MULTIFACTOR MACROECONÓMICO}

El modelo multifactor se estima en series de tiempo mediante MCO y la matriz de White para la detección de errores estándar robustos. Además, con el fin de comprobar su solidez, se someten las variables seleccionadas y el modelo estimado a diversas pruebas estadísticas a partir de la formulación de un conjunto de hipótesis que permitan comprobar su calidad para poder llevar a cabo la descripción de la inferencia estadística correctamente. La hipótesis que se busca probar con el modelo multifactor aplicado a los hedge funds es: "Las sorpresas (componentes

\footnotetext{
${ }^{26}$ El criterio empleado para seleccionar el número de factores fue el de la raíz latente, es decir, se seleccionaron sólo los factores que explican más de 5\% de la varianza. Este mismo criterio es empleado por Gómez-Bezares, Madariaga y Santibáñez (1994).

${ }^{27}$ En las matrices de factores los hedge funds son ordenados de acuerdo al tamaño de su correlación con los factores (en valor absoluto).

${ }^{28}$ Los resultados de la estimación y los contrastes de especificación y diagnóstico de los modelos utilizados para calcular la inflación esperada y el residual del mercado pueden solicitarse a la autora para su consulta.
} 


\section{Cuadro 2. Tipos de riesgo macroeconómico, definiciones y alteraciones macroeconómicas (continúa)}

\begin{tabular}{|c|c|c|}
\hline $\begin{array}{l}\text { Tipo de riesgo } \\
\text { macroeconómico }\end{array}$ & Definición & Sorpresa macroeconómica \\
\hline $\begin{array}{l}\text { Riesgo de } \\
\text { inflación }\end{array}$ & $\begin{array}{l}\text { Combinación de los com- } \\
\text { ponentes no esperados de } \\
\text { las tasas de inflación. }\end{array}$ & $\begin{array}{l}\text { Inflación no esperada }\left(N E I n f_{t}\right) \text { : es la diferen- } \\
\text { cia entre la inflación observada y la infla- } \\
\text { ción esperada: } N E \operatorname{lnf} f_{t}=\operatorname{lnf} f_{t}-E \operatorname{lnf} f_{t}\end{array}$ \\
\hline $\begin{array}{l}\text { Riesgo del ciclo } \\
\text { económico }\end{array}$ & $\begin{array}{l}\text { Cambio no esperado en el } \\
\text { nivel de la actividad eco- } \\
\text { nómica. }\end{array}$ & $\begin{array}{l}\text { Crecimiento de la producción industrial (IPI- } \\
\left.M_{t}\right) \text { : es el cambio mensual en el logaritmo } \\
\text { natural del índice de producción industrial: } \\
\qquad I P I M_{t}=\ln \left\{\left[\left(\left.I P\right|_{t} /\left.I P\right|_{t-1}\right)-1\right] * 100\right\}\end{array}$ \\
\hline $\begin{array}{l}\text { Riesgo en la } \\
\text { confianza }\end{array}$ & $\begin{array}{l}\text { Cambio no esperado en la } \\
\text { voluntad del inversor para } \\
\text { emprender inversiones re- } \\
\text { lativamente riesgosas. }\end{array}$ & $\begin{array}{l}\text { Default premium }\left(D P_{t}\right) \text { : es la diferencia en- } \\
\text { tre la rentabilidad mensual de los bonos de } \\
\text { las empresas de baja calificación y la renta- } \\
\text { bilidad mensual de los bonos del gobierno } \\
\text { estadounidense a largo plazo: } \\
D P_{t}=B a a_{t}-G L P_{t}\end{array}$ \\
\hline $\begin{array}{l}\text { Riesgo de } \\
\text { horizonte de } \\
\text { tiempo }\end{array}$ & $\begin{array}{l}\text { Cambio no anticipado de } \\
\text { los deseos del inversor en } \\
\text { los tiempos para realizar } \\
\text { pagos. }\end{array}$ & $\begin{array}{l}\text { Term structure }\left(T S_{t}\right) \text { : es la diferencia entre la } \\
\text { rentabilidad mensual de los bonos del go- } \\
\text { bierno norteamericano a largo plazo y la } \\
\text { rentabilidad mensual de los bonos Trea- } \\
\text { sury-bill del gobierno estadounidense a un } \\
\text { mes: } T S_{t}=G L P_{t}-T \text { tbill } t\end{array}$ \\
\hline $\begin{array}{l}\text { Riesgo de } \\
\text { tiempo de } \\
\text { mercado }\end{array}$ & $\begin{array}{l}\text { Se calcula como la parte } \\
\text { de la rentabilidad de un } \\
\text { índice de renta variable } \\
\text { que no está explicado por } \\
\text { los primeros cuatro tipos } \\
\text { de riesgo macroeconómico } \\
\text { y un término intercepto. }\end{array}$ & $\begin{array}{l}\text { Residual del mercado }(S \& P) \text { : Es la parte del } \\
\text { S\&P500 real }(S \& P=S \& P 500-T b i l l) \text { que el resto } \\
\text { de los factores de riesgo seleccionados no } \\
\text { son capaces de explicar, es decir, el residual } \\
\left(u_{t}\right) \text { en la ecuación: } S \& P_{t}=b_{0}+b_{1} N E I n f_{t}+b_{2} \\
I P I M_{t}+b_{3} D P_{t}+b_{4}+T S_{t} u_{t}\end{array}$ \\
\hline
\end{tabular}

*Fuente: Elaboración propia a partir de Chen, Roll y Ross (1986); Berry, Burmeister y McElroy (1988); Connor (1995), y Burmeister, Roll y Ross (2003).

no esperados) macroeconómicas [NEInf, IPIM, DP, TS y $S \& P$ ] que influyen en la rentabilidad de las diferentes clases de activos tienen poder explicativo sobre los índices de rentabilidad de los hedge funds". Dada esta hipótesis el modelo se expresa como:

donde:

$$
\stackrel{\prime}{I} R_{j i t}=\beta_{0}+\beta_{j i 1} N E I n f+\beta_{j i 2} D P+\beta_{j i 3} T S+\beta_{j i 4} I P I M+\beta_{j i 5} S \& P+\varepsilon_{j i t},
$$

$I R_{i t}$ es el índice $j$ que contiene la información sobre la rentabilidad de los hedge funds que siguen la estrategia $i$ en el mes $t$. 
$\beta_{0}$ es el término constante.

$\beta_{j i 1}, \beta_{j i 2}, \beta_{j i 3}, \beta_{j i 4}, \mathrm{y} \beta_{j i 5}$ son los riesgos de exposición del índice $j$ que contiene la información sobre la rentabilidad de los hedge funds que siguen la estrategia $i$ hacia las $F$ sorpresas macroeconómicas.

[NEInf, IPIM, DP, TS y S\&P] son las $F$ sorpresas macroeconómicas en el mes $t$. $\varepsilon_{j i t}$ es el término error, donde:

$j=1, \ldots, 72 ; i=1, \ldots, 9 ; \mathrm{y} t=1, \ldots, 54$ para los hedge funds activos.

$j=1, \ldots, 19 ; i=1, \ldots, 8 ; \mathrm{y} t=1, \ldots, 45$ para los hedge funds liquidados.

$F=1, \ldots, 5$ para los hedge funds activos y para los hedge funds liquidados.

El modelo tiene por objetivo comprobar si las sorpresas macroeconómicas tienen una influencia estadísticamente significativa sobre los índices que contienen información acerca de la rentabilidad de los hedge funds o si, por el contrario, carecen de influencia estadísticamente significativa.

\section{Resultados del análisis empírico}

En esta sección se reportan los resultados de la estimación del modelo multifactor. En primer lugar, se exponen los contrastes de especificación y diagnóstico necesarios para la comprobación de la solidez del modelo. En seguida, se presenta la inferencia estadística por cada estrategia seguida.

En el cuadro 3 se exponen los sumarios estadísticos de las sorpresas macroeconómicas seleccionadas como variables explicativas del modelo multifactor. ${ }^{29}$ En el cuadro 4 se presentan las matrices de correlación de las sorpresas macroeconómicas. La correlación entre la sorpresa residual del mercado y el resto es nula por la manera en la que fue calculada dicha sorpresa. ${ }^{30}$ También se observa que la correlación entre el default premium y la term structure es más alta que el resto, lo cual no es de extrañar ya que en el cálculo de ambas se emplea la rentabilidad mensual de los bonos del gobierno estadunidense a largo plazo. Sin embargo, una no sustituye a la otra debido a que capturan diferentes movimientos en los precios. ${ }^{31}$ Además, el nivel de correlación entre ellas no es representativo de problemas de multicolinealidad.

\footnotetext{
${ }^{29}$ Los sumarios estadísticos, las matrices de correlación y los análisis de regresión en series temporales fueron realizados con el paquete estadístico de cómputo Econometric Views 5.

${ }^{30}$ Es la parte de la rentabilidad real del S\&P500 no explicada por el resto de las sorpresas.

${ }^{31}$ Chen, Roll y Ross (1986) también se encontraron con esta situación y continuaron utilizando ambas variables.
} 


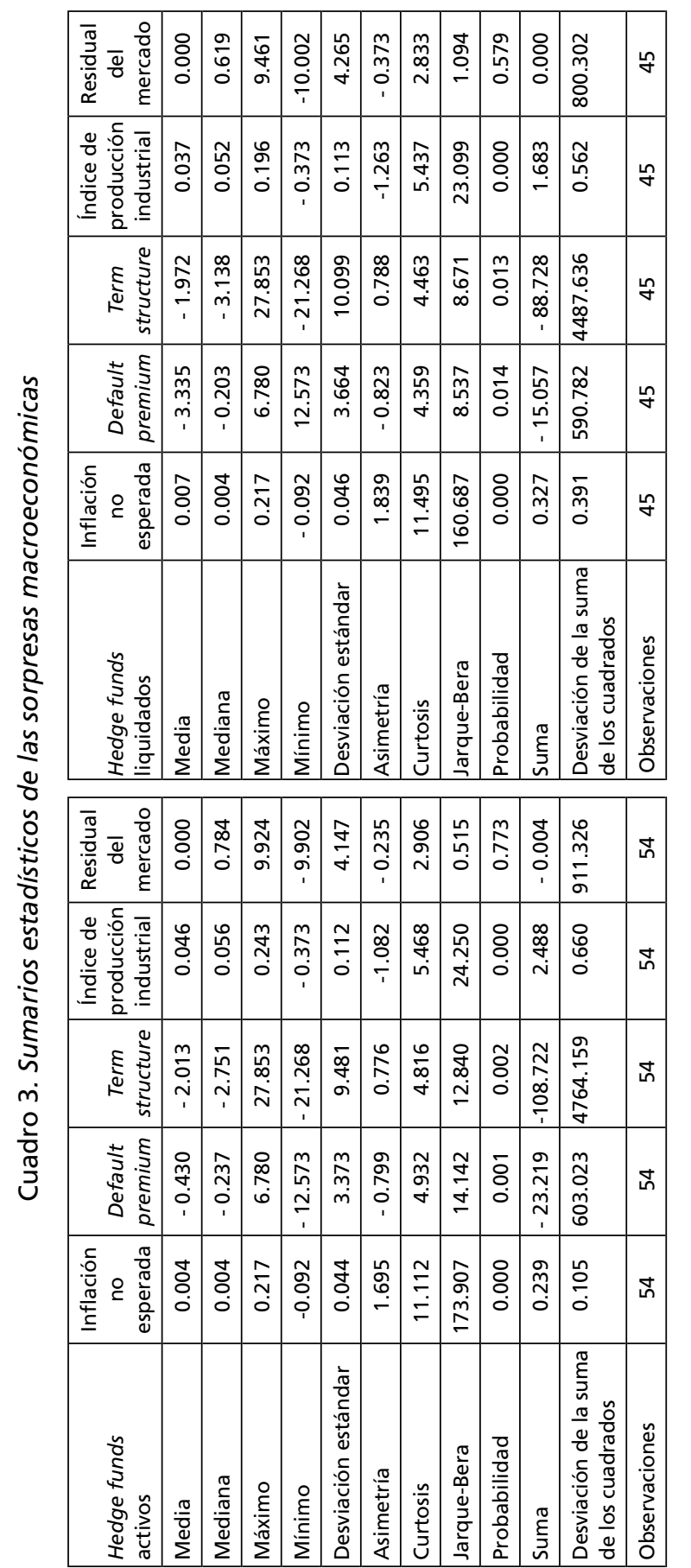

\begin{tabular}{|c|c|c|c|c|c|}
\hline 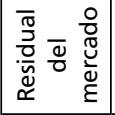 & \begin{tabular}{l}
0 \\
\hdashline \\
0
\end{tabular} & $\begin{array}{l}8 \\
\vdots \\
0\end{array}$ & 웅 & \& & $\underset{-}{\stackrel{0}{-}}$ \\
\hline 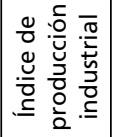 & 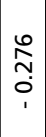 & $\begin{array}{l}0 \\
\stackrel{0}{0} \\
0 \\
1\end{array}$ & $\begin{array}{l}\stackrel{0}{\tilde{O}} \\
\dot{0}\end{array}$ & $\stackrel{\circ}{\circ}$ & $\begin{array}{l}8 \\
0 \\
0\end{array}$ \\
\hline 0 & & & & & \\
\hline
\end{tabular}

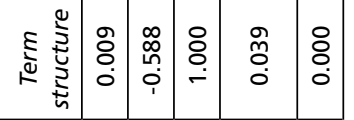

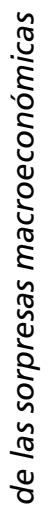

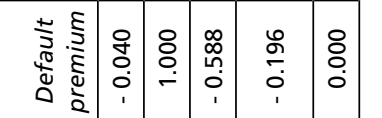

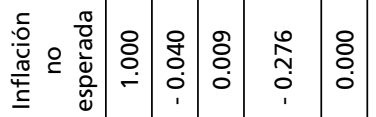

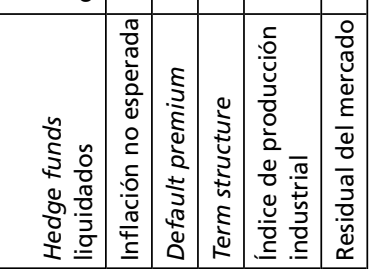

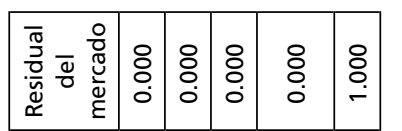

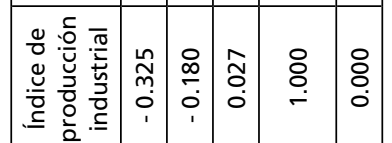

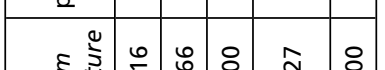

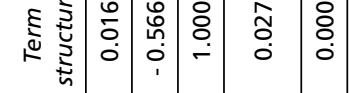

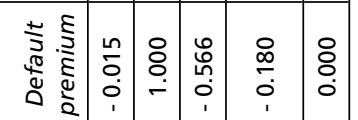

\begin{tabular}{|c|c|c|c|c|c|}
\hline 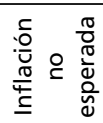 & $\underset{\leftrightarrow}{\stackrel{\Delta}{ }}$ & $\begin{array}{l}\frac{1}{2} \\
0 \\
0\end{array}$ & $\begin{array}{l}0 \\
0 \\
0 \\
0\end{array}$ & $\begin{array}{l}\stackrel{\sim}{n} \\
\text { ñ } \\
0\end{array}$ & : \\
\hline 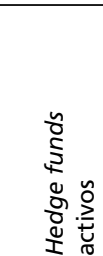 & 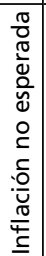 & 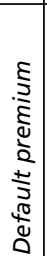 & 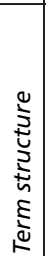 & 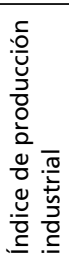 & 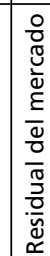 \\
\hline
\end{tabular}




\section{a) Contrastes de especificación y diagnóstico}

El supuesto implícito en el cual se basa cualquier análisis de regresión que considera series de tiempo es que son estacionarias. Por ello, se aplicó como prueba de raíz unitaria la de Dickey-Fuller aumentada, para comprobar si las sorpresas macroeconómicas y los índices de rentabilidad empleados son estacionarios. Como era de esperarse -por la forma en la que se construyeron las variables- $-{ }^{32}$ los resultados confirman la estacionariedad de todas, ${ }^{33}$ ya que el valor del estadístico $\tau$ siempre es superior al valor crítico de MacKinnon en $99 \%$ de confianza y, por tanto, se rechaza la hipótesis nula de no estacionariedad.

Tras confirmar la existencia de estacionariedad, estimamos el modelo multifactor con cada uno de los índices de rentabilidad de los hedge funds activos y liquidados para comprobar si los factores de riesgo macroeconómico influyen en su rentabilidad. Por tanto, estimamos el modelo mediante MCO para cada uno de los índices sin ajustes de un modelo $\operatorname{AR}(p)$, un $\operatorname{MA}(q)$ o un $\operatorname{ARMA}(p, q)$, con el fin de comprobar si los residuos de cada una de las regresiones son estacionarios. El cuadro 5 presenta los resultados de los coeficientes estimados sin ajustes $^{34}$ y las pruebas de raíz unitaria aplicadas a los residuos de cada una de las regresiones de los índices de rentabilidad de los hedge funds liquidados. ${ }^{35}$

En las pruebas de raíz unitaria aplicadas se rechaza la hipótesis nula de no estacionariedad, ya que en todos los casos el valor del estadístico $\tau$ es superior al valor crítico en $99 \%$ de confianza. Por ende, los resultados de estas pruebas

${ }^{32}$ Los índices de rentabilidad son estacionarios debido a que se trata de los factores obtenidos mediante el análisis factorial, los cuales tienen media 0 y varianza 1 , que es la condición necesaria para la estacionariedad. De igual forma, la manera en la que se construyeron las sorpresas macroeconómicas eliminó el movimiento de tendencia, que es una característica común de las series macroeconómicas.

${ }^{33}$ Los resultados favorables de los contrastes de estacionariedad pueden solicitarse a la autora para su consulta.

${ }^{34}$ Aunque las regresiones del cuadro 5 pueden presentar problemas de heterocedasticidad y autocorrelación, la prueba de Dickey-Fuller aumentada requiere que el modelo no tenga ningún ajuste para poder determinar si los residuos son estacionarios o no, ya que el análisis de causalidad supone que las series de tiempo involucradas son estacionarias. Por ello, las pruebas de estacionariedad deben efectuarse antes que las de causalidad.

${ }^{35}$ Debido a que se utilizaron 72 índices de rentabilidad para el análisis de los hedge funds activos, la tabla con los coeficientes estimados sin ajustes y las pruebas de raíz unitaria es de grandes dimensiones, por lo que únicamente se presentan los resultados de los hedge funds liquidados. Los resultados de primeros pueden solicitarse a la autora para su consulta, no obstante, fueron favorables en todos los casos. 
confirman la estacionariedad de los residuos obtenidos en la estimación del modelo multifactor para cada uno de los índices de rentabilidad de los hedge funds, activos y liquidados. Esto indica que las regresiones en series temporales realizadas son válidas y es posible continuar con el análisis. Se comienza con los contrastes básicos de White y Breusch-Godfrey para identificar problemas de heterocedasticidad y autocorrelación, respectivamente. Posteriormente se aplican contrastes de Jarque-Bera y de Ramsey para la comprobación de normalidad de las perturbaciones y linealidad del modelo, respectivamente.

El cuadro 6 presenta los coeficientes estimados del modelo multifactor aplicado a cada uno de los índices de rentabilidad de los hedge funds liquidados. ${ }^{36}$ En él se puede comprobar que después de realizar los ajustes necesarios con un modelo $\operatorname{AR}(p)$, un $\operatorname{MA}(q)$ o un $\operatorname{ARMA}(p, q)$, en ningún caso se rechazan las hipótesis nulas de ausencia de autocorrelación y de ausencia de heterocedasticidad, ya que la probabilidad respectiva es superior a $0.05 \mathrm{y}$, por tanto, las perturbaciones de todas las regresiones son homocedásticas y están serialmente incorrelacionadas.$^{37} \mathrm{La}$ situación anterior ocurre también en todas las regresiones de los índices de rentabilidad de los hedge funds activos. Las pruebas secundarias de normalidad de las perturbaciones y linealidad del modelo son superadas con éxito en $84 \%$ y $95 \%$ de las regresiones, respectivamente, para los índices de rentabilidad de los hedge funds liquidados, mientras que para los índices de los activos son superados con éxito en $83 \%$ y $97 \%$ de las regresiones, respectivamente. ${ }^{38}$

\section{b) Análisis de los hedge funds por estrategia seguida}

La descripción de los resultados en esta sección se basa en las correlaciones encontradas entre los índices de rentabilidad de los hedge funds activos y las mis-

${ }^{36}$ Por el mismo motivo que en la nota 35 , sólo se presentan los coeficientes estimados con ajustes y los contrastes de especificación y diagnóstico para los hedge funds liquidados. Los resultados de los hedge funds activos, pueden solicitarse a la autora para su consulta, aunque también fueron favorables en todos los casos.

${ }^{37}$ Los ajustes con un modelo $\operatorname{AR}(p)$, un $\operatorname{MA}(q)$ o un $\operatorname{ARMA}(p, q)$ se llevan a cabo si en los resultados de los contrastes de heterocedasticidad y autocorrelación se determina que son necesarios.

${ }^{38}$ En tres de las 19 regresiones de los índices de los hedge funds liquidados y en 12 de las 72 regresiones de los índices de los activos se rechaza la hipótesis nula de normalidad debido a que la probabilidad es inferior a 0.05 . No obstante, el cumplimiento de esta hipótesis no es necesaria para la obtención de los estimadores de MCO, ya que las propiedades de estos estimadores se siguen conociendo aun cuando no se cumpla este supuesto. Por otro lado, en una de las 19 regresiones de los índices de fondos liquidados y en dos de las 72 regresiones de los índices de los activos se rechaza la hipótesis nula de linealidad del modelo debido a que la probabilidad es inferior a 0.05 . 


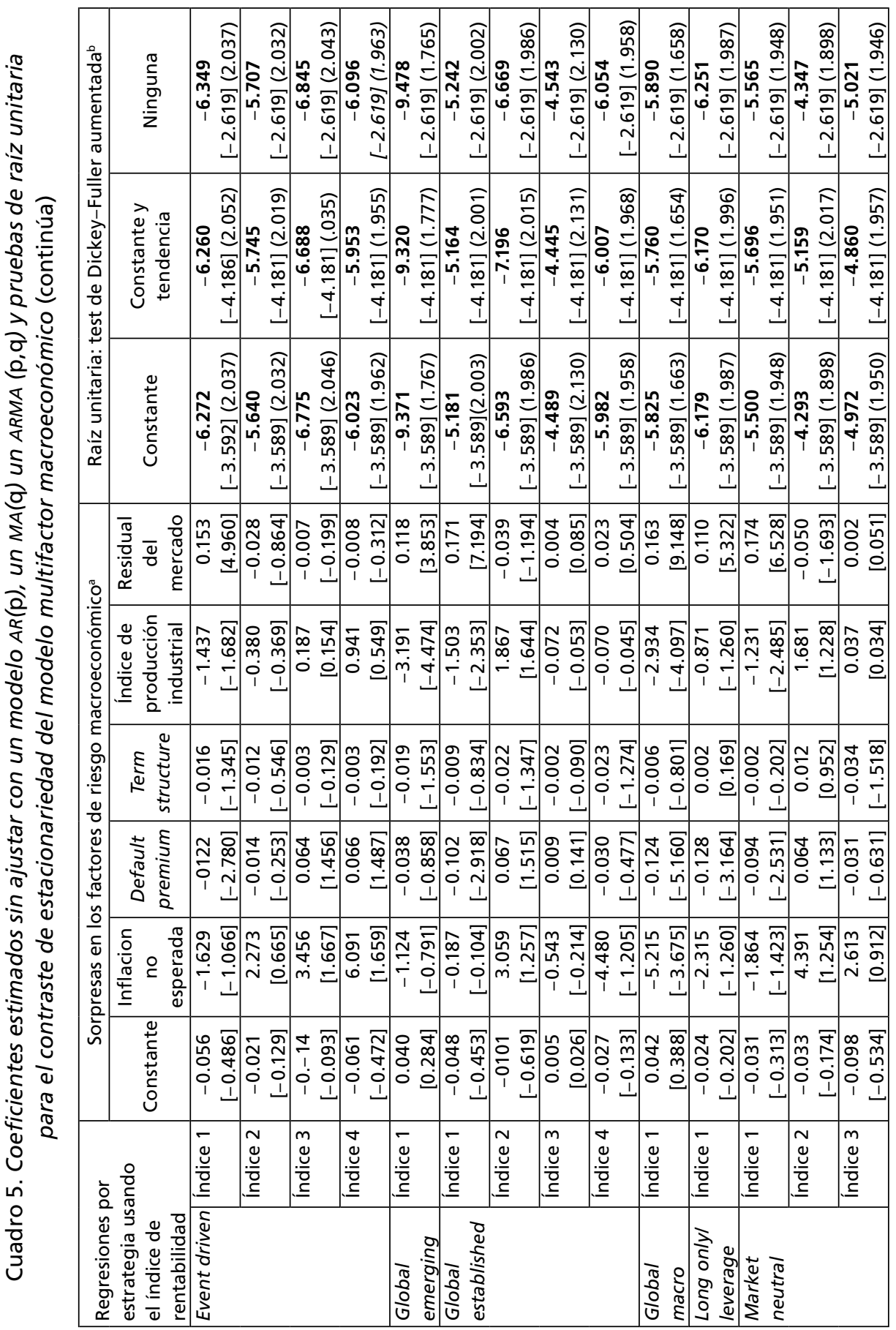




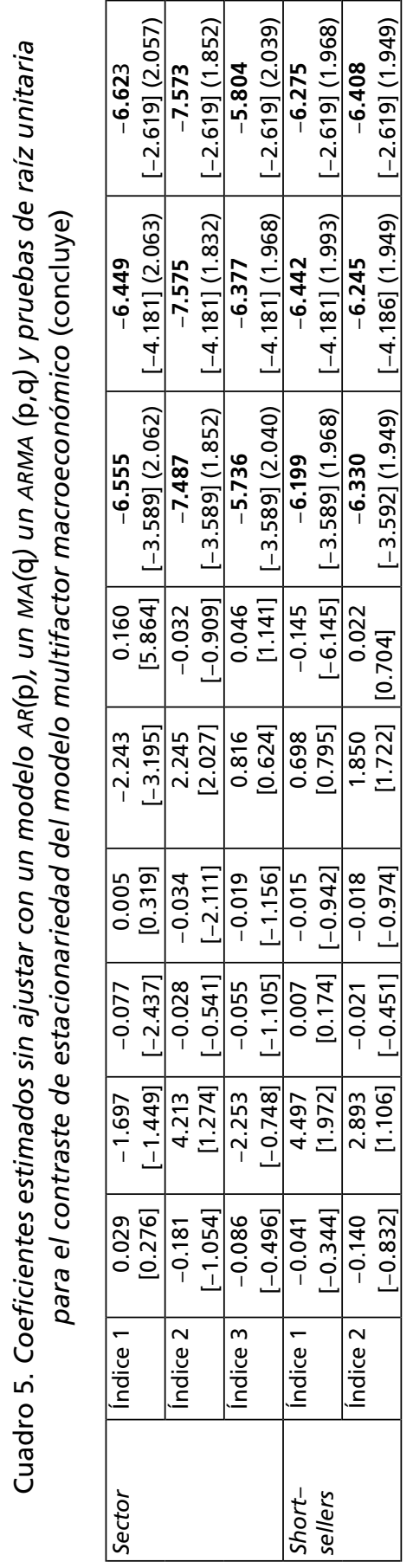

\begin{tabular}{|c|c|c|c|c|c|c|}
\hline & $\begin{array}{c}\text { Heterocedasticidad: } \\
\text { prueba de }^{\text {White }}\end{array}$ & 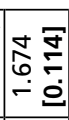 & 今. & 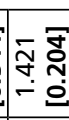 & $\begin{array}{ll}\sim & \\
2 & 0 \\
0 & 0 \\
0 & 0 \\
0 & 0\end{array}$ & 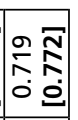 \\
\hline 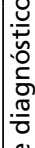 & $\begin{array}{l}\text { Autocorrelación: } \\
\text { prueba LM de } \\
\text { Breusch-Godfrey }\end{array}$ & $\begin{array}{ll}\bar{m} & \bar{\sigma} \\
6 & 0 \\
0 & 0 \\
0\end{array}$ & 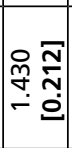 & $\begin{array}{ll}= & \frac{J}{\sigma} \\
0 & \infty \\
0 & 0 \\
0\end{array}$ & 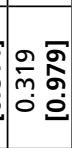 & \\
\hline $\mathscr{a}$ & $\begin{array}{l}\text { Normalidad: } \\
\text { prueba de } \\
\text { Jarque-Bera }\end{array}$ & 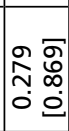 & $\mid \begin{array}{ll}\hat{b} & \overline{5} \\
0 & 0 \\
\forall & 0 \\
\forall\end{array}$ & {$\left[\begin{array}{ll}- & \infty \\
m & \vdots \\
m & \infty \\
0 & 0\end{array}\right.$} & 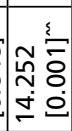 & 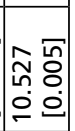 \\
\hline 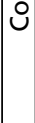 & $\begin{array}{l}\text { Linealidad: RESET } \\
\text { de Ramsey }\end{array}$ & 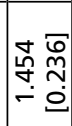 & 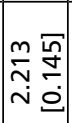 & {$\left[\begin{array}{ll}- & 0 \\
0 & \infty \\
n & 0 \\
0 & 0 \\
0 & 0\end{array}\right.$} & 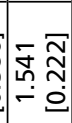 & $\left|\begin{array}{cc}m & \bar{n} \\
\infty & n \\
\infty & n \\
0 & 0 \\
0\end{array}\right|$ \\
\hline o & Dur & 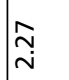 & $\mathbb{N}$ & స్త & 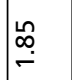 & గొ \\
\hline$\stackrel{-\frac{1}{0}}{0}$ & $R^{2}$ ajus & 爷 & $\stackrel{\circ}{\circ}$ & $\stackrel{\circ}{m}$ & $\stackrel{\circ}{m}$ & $m$ \\
\hline w & $\tilde{q}$ & 今̊ & $\stackrel{8}{8}$ & ने & 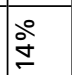 & \\
\hline & 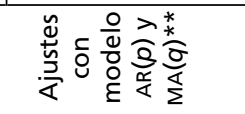 & & ' & ' & ' & \\
\hline \% & 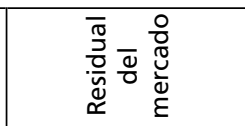 & 范 & $\mid \begin{array}{cc}\infty & 7 \\
0 & 0 \\
0 & 0 \\
0 & 0 \\
1 & 1\end{array}$ & $\mid \begin{array}{l}2 \\
8 \\
0 \\
0 \\
0\end{array}$ & {$\left[\begin{array}{ll}\infty & \frac{\pi}{2} \\
0 & 0 \\
0 & 0 \\
0 & 0 \\
1 & 1\end{array}\right.$} & 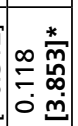 \\
\hline סू & $\begin{array}{l}\text { indice de } \\
\text { producción } \\
\text { industrial }\end{array}$ & 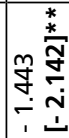 & $\begin{array}{cc}0 \\
0 & 0 \\
0 & 0 \\
m & m \\
0 & 0 \\
1 & 1\end{array} \mid$ & $\frac{\pi}{\infty}$ & 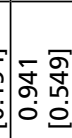 & 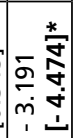 \\
\hline 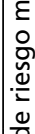 & 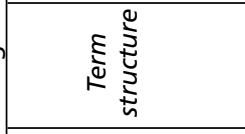 & 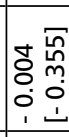 & $\mid$\begin{tabular}{cc}
2 & 0 \\
\hdashline & 1 \\
0 & 0 \\
0 & 0 \\
1 & 1
\end{tabular} & 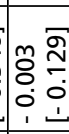 & $\mid \begin{array}{ll}m & \bar{\pi} \\
0 & 0 \\
0 & 0 \\
0 & 0 \\
1 & 1\end{array}$ & 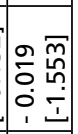 \\
\hline $\begin{array}{l}\tilde{y} \\
\tilde{\omega}\end{array}$ & 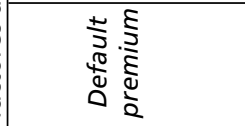 & 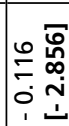 & $\begin{array}{ll}0 & \bar{n} \\
0 & 0 \\
0 & 0 \\
0 & 0 \\
0 & 1\end{array}$ & 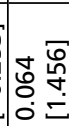 & : & {$\left[\begin{array}{ll}\infty & 0 \\
0 & 0 \\
0 & 0 \\
0 & 0 \\
0 & 0 \\
1 & 1\end{array}\right.$} \\
\hline o & 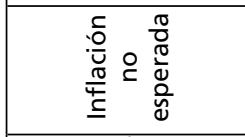 & 总 & $\begin{array}{ll}m & 0 \\
& 0 \\
& 0 \\
& 0\end{array}$ & 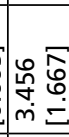 & 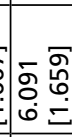 & \\
\hline$\sim$ & 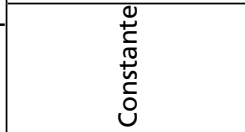 & $\mid \begin{array}{ll}0 & \overline{1} \\
0 & 0 \\
0 & 0 \\
0 & 0 \\
1 & 0 \\
1 & 1\end{array}$ & $\left|\begin{array}{ll}\bar{N} & \overline{2} \\
0 & - \\
0 & 0 \\
1 & 1\end{array}\right|$ & $\mid \begin{array}{ll}0 & m \\
0 & 0 \\
0 & 0 \\
0 & 0 \\
1 & 1\end{array}$ & $\mid \begin{array}{ll}- & \overline{8} \\
0 & \frac{f}{f} \\
0 & 0 \\
1 & 1\end{array}$ & $\begin{array}{ll}0 \\
0 \\
0 & \stackrel{0}{2} \\
0 & 0\end{array}$ \\
\hline & 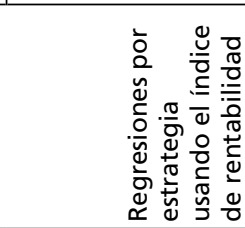 & 造 - & 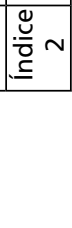 & $\frac{\mathscr{U}}{\overline{0}} \mathrm{~m}$ & 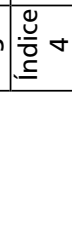 & 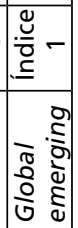 \\
\hline
\end{tabular}




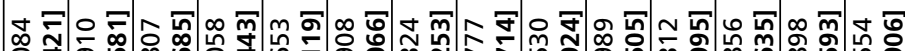

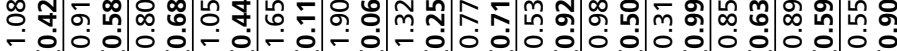

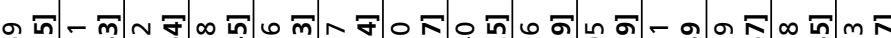

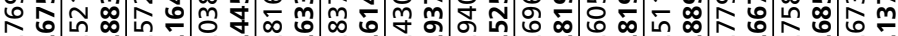

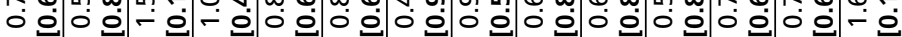

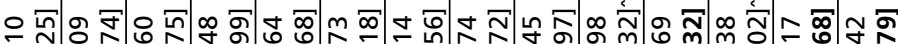

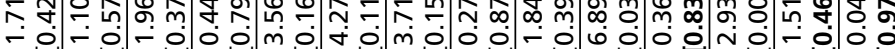

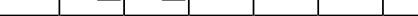

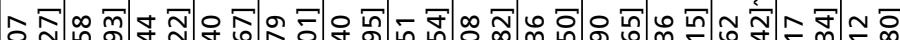
슨 o 过

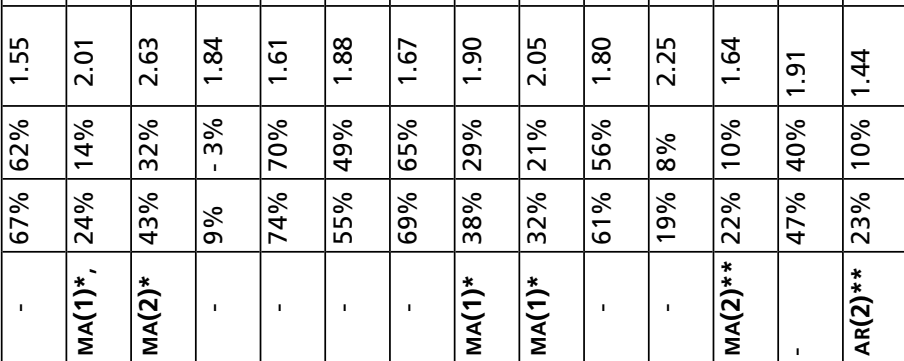

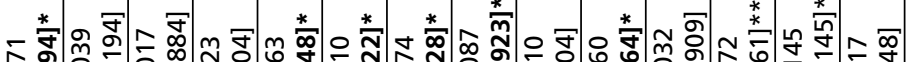
₹

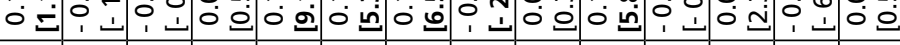

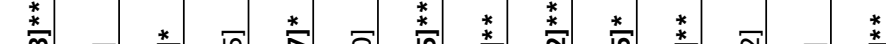

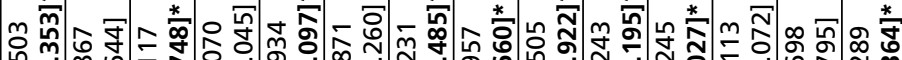
I

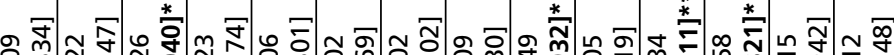
‡

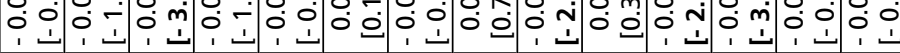
草

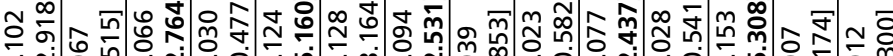
on no no -

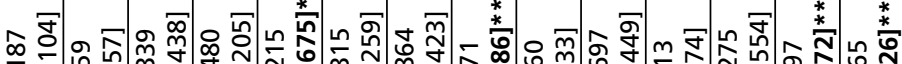
¡o

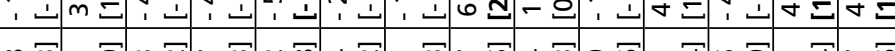

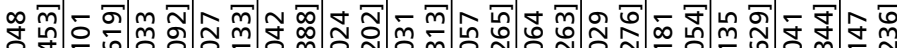

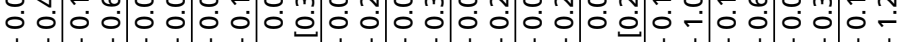

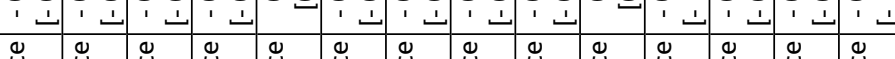

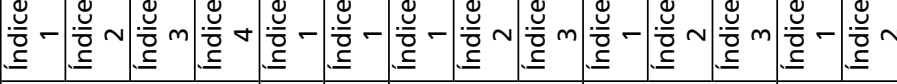

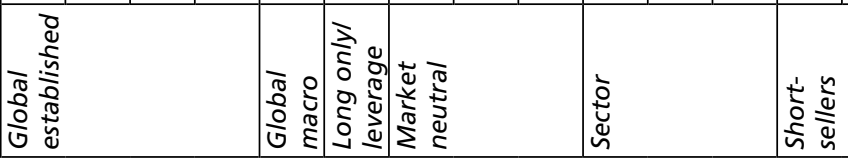

نั

$\dot{y}$ ठ

经

응 은

웅

ㅁำ

\% 政

* * 는

$>$ 응

웡

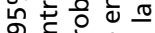

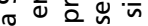

$*$ *

$\pi \cdot \frac{0}{0} \frac{\pi}{\pi}$

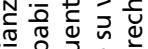

进 넝

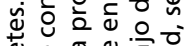

迸

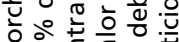

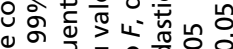

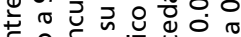

ฯ $ᄋ \frac{0}{4}$

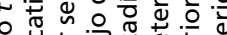

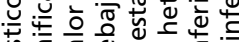

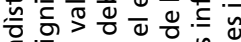

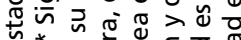

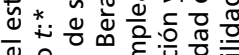

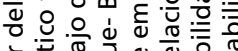

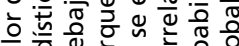

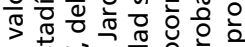

ब苟山定

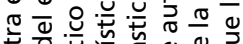

눙

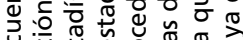

ब

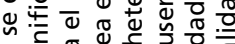

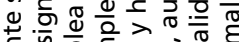

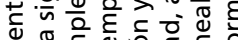

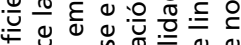

吨

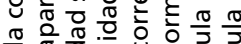

응

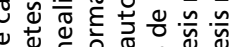

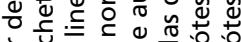

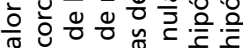

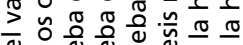

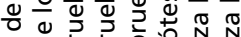

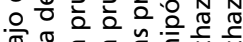

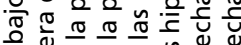

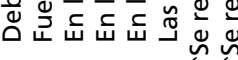


mas sorpresas macroeconómicas ${ }^{39}$ mostradas en el cuadro 6 para los liquidados, y en la bondad de ajuste de cada modelo. Para una mayor claridad y sencillez en la exposición, conviene destacar que durante la extracción de factores el método de ejes principales identifica los fondos con características similares $\mathrm{y}$, por lo tanto, favorece las correlaciones entre ellos, de modo que el primer factor extraído se contempla como el que mejor resume las relaciones lineales que manifies$\tan$ los hedge funds. Por lo anterior, las relaciones encontradas entre los índices 1 y las sorpresas macroeconómicas (por estrategia seguida) se exponen a detalle a continuación.

Cabe señalar que el análisis de cada estrategia comienza con una breve descripción de ella, con el propósito de destacar sus aspectos fundamentales y la relación principal que tiene con las sorpresas macroeconómicas. ${ }^{40}$ Sin embargo, debido a la relevancia de la inflación no esperada y de la sorpresas residual del mercado en el sector de los hedge funds, la mayor parte de las relaciones encontradas se describe al final del análisis por estrategia seguida.

Event driven. Las subestrategias consideradas dentro de la estrategia event driven (entre ellas risk arbitrage, merger arbitrage y distressed arbitrage) se caracterizan por buscar situaciones especiales y oportunidades para capitalizarse a partir de los movimientos en los precios causados por eventos corporativos significativos, como reorganizaciones, fusiones, adquisiciones, reestructuraciones, suspensiones de pagos (insolvencia) y quiebras. Los gestores que siguen esta estrategia invierten en la deuda de las empresas en problemas en lugar de comprar sus acciones, ya que en caso de quiebra los acreedores tienen prioridad sobre los accionistas. No obstante, si la empresa sale a flote, la deuda de la empresa en problemas (comprada por los hedge funds a precio reducido) se revalorizará y

\footnotetext{
${ }^{39}$ Los resultados relativos a los hedge funds activos pueden solicitarse a la autora para su consulta. No obstante, en esta sección se describen las correlaciones encontradas entre los índices de rentabilidad 1 de los hedge funds activos y las sorpresas macroeconómicas, por estrategia seguida.

${ }^{40}$ López-Pascual y Camarero A. (2013) analizan el perfil de riesgo de diferentes estrategias de inversión seguidas por los hedge funds. Utilizando los índices de rentabilidad mensual por estrategia seguida proporcionados por la Hedge Fund Research (HFRI), de junio de 2007 a marzo de 2011, señalan que el análisis de la rentabilidad histórica y del comportamiento estadístico de las estrategias proporciona una importante fuente de información para evaluar y comprender las estrategias de inversión de los hedge funds. Argumentan que en este negocio las altas rentabilidades en exceso obtenidas en un punto específico del ciclo económico podrían volverse sistemáticamente inferiores una vez que se producen cambios en el ciclo. Por tanto, la rentabilidad de un hedge fund debe entenderse de acuerdo al contexto de la estrategia utilizada y del ciclo económico.
} 
será del interés de otros agentes financieros, lo que generará altas plusvalías para estos hedge funds. Por lo anterior, la principal sorpresa macroeconómica la que deben poner especial atención los fondos que recurren a la event driven es el default premium, ya que ésta considera el tipo de interés ofrecido por las empresas de baja calificación. Los resultados muestran que en la regresión del modelo multifactor con el índice de rentabilidad $1^{41}$ se obtuvieron coeficientes negativos y estadísticamente significativos para dicha sorpresa, lo que indica que cuando este diferencial disminuye, aumenta la rentabilidad de los fondos que usan la event driven. Una posible interpretación sería que la empresa en dificultades a la que los hedge funds le compraron su deuda ha logrado salir a flote y se ha vuelto atractiva de nuevo para los fondos tradicionales y para los inversores institucionales, proporcionado altas rentabilidades a los hedge funds que compraron la deuda a precio de ganga. ${ }^{42}$

Global emerging. Los gestores que siguen la estrategia global emerging invierten en los mercados financieros menos maduros del mundo por la alta rentabilidad que éstos ofrecen. El centro de atención son las economías emergentes ubicadas en Asia, América Latina, Medio Oriente, Rusia y Europa del Este, donde asumen posiciones largas y cortas en acciones de las principales empresas ${ }^{43}$ y en deuda de estos gobiernos. Debido a que en este tipo de economías las ventas al descubierto no están permitidas así como, tampoco algunos otros productos derivados más sofisticados, los fondos que usan la global emerging a menudo deben cambiar de mercado cuando las valorizaciones en renta fija y renta variable de las economías emergentes hacen que las posiciones largas sean poco atractivas. Con base en esta descripción, queda claro que las sorpresas macroeconómicas default premium (que involucra la deuda de las empresas), term structure

${ }^{41}$ Para los hedge funds liquidados, el índice de rentabilidad 1 agrupa 58\% de los factores comunes que explican las interrelaciones entre los fondos que siguen la estrategia event driven. Mientras que en el caso de los activos con esta misma estrategia, el índice 1 agrupa 48\%, 49\% y 44\%, en cada uno de los tres grupos, respectivamente. Además, se observa que la regresión con el índice 1 para los hedge funds liquidados que siguen la event driven tiene una bondad de ajuste de $64 \%$, mientras que para los activos es de $75 \%, 72 \%$ y $58 \%$, respectivamente.

${ }^{42} \mathrm{Si}$ la reestructuración de una empresa de baja calificación y las medidas que su administración haya adoptado consiguieron hacerla reflotar, ya no se verá obligada a pagar un tipo de interés de largo plazo mayor que el de los bonos del gobierno de largo plazo y, por tanto, el diferencial entre ambos disminuirá.

${ }^{43}$ En los mercados de estas economías suelen cotizar muy pocas empresas (las más grandes del país). 
(que implica los tipos de interés de los bonos gubernamentales) y residual del mercado juegan un papel muy importante en la rentabilidad de estos hedge funds. Los resultados muestran que en la regresión con el índice $1^{44}$ para estos hedge funds se obtuvieron coeficientes positivos y estadísticamente significativos en la sorpresa residual del mercado, lo que indica que estos fondos apuestan por la evolución positiva del mercado global. Por otra parte, las sorpresas default premium y term structure obtuvieron coeficientes negativos y estadísticamente significativos en la regresión con el índice 1 del primer y segundo grupo de hedge funds activos que aplican la global emerging. Este resultado sugiere que cuando aumenta el costo de oportunidad de inversión en economías en vías de desarrollo, disminuye la rentabilidad de los fondos que siguen esta estrategia, ya que han realizado inversiones relativamente riesgosas que no se están viendo compensadas. $^{45}$

Global established. Al contrario de la estrategia anterior, con la global established, los hedge funds buscan principalmente oportunidades de inversión en renta variable en mercados maduros, como el europeo, el japonés y el estadounidense. Asumen posiciones largas y cortas con el fin de explotar las oportunidades de inversión existentes debido a las discrepancias presentes en los precios de títulos similares (por ejemplo, acciones de una empresa estadounidense frente a acciones de una empresa europea, ambas del mismo sector). Sin embargo, estas discrepancias por lo regular son pequeñas, lo que obliga a los gestores a asumir un mayor apalancamiento. Esta estrategia no es neutral al mercado, ya que al tomar posiciones más largas o más cortas están apostando por un sesgo alcista o a la baja en su posición. Los resultados muestran que en la regresión con el índice $1^{46}$

${ }^{44}$ Para los hedge funds liquidados que aplican la global emerging, el índice de rentabilidad 1 agrupa $64 \%$ de los factores comunes que explican las interrelaciones entre los fondos que siguen esta estrategia. Mientras que en los dos grupos de los activos, el índice 1 agrupa 48\% y 55\%, respectivamente. Además, se observa que la regresión con el índice 1 para los hedge funds liquidados que siguen la global emerging tiene una bondad de ajuste de $41 \%$, mientras que para los activos es de $40 \%$ y $52 \%$, respectivamente.

${ }^{45}$ La mayoría de los activos tiene una exposición positiva al riesgo en la confianza (medido indirectamente por la sorpresa default premium). Sin embargo, los pequeños (en este caso representados por los activos del mercado monetario de las economías emergentes) generalmente presentan una mayor exposición al riesgo en la confianza que los grandes (presentes en las economías desarrolladas).

${ }^{46}$ Para los hedge funds liquidados de estrategia global established, el índice de rentabilidad 1 agrupa 39\% de los factores comunes que explican las interrelaciones entre lo fondos que siguen esta estrategia. A su vez, para los activos, el índice 1 agrupa 54\%, 48\%, 47\% y 48\% en cada uno de 
para los hedge funds, tanto activos como liquidados, se obtuvieron coeficientes positivos y estadísticamente significativos para la sorpresa residual del mercado. Esto confirma que se trata de fondos oportunistas que aprovechan situaciones puntuales o tendencias en los mercados. Con esta misma regresión, los hedge funds activos y liquidados obtuvieron coeficientes negativos y estadísticamente significativos ante la sorpresa default premium; es decir, cuando este diferencial aumenta, la rentabilidad de estos fondos disminuye. Por otro lado, la sorpresa term structure sólo es estadísticamente significativa en las regresiones de los índices de rentabilidad que agrupan un porcentaje pequeño de los factores comunes que explican las interrelaciones entre los hedge funds que siguen la global established.

Global international. Los gestores de hedge funds que se conducen con la estrategia global international ponen mucha atención a los cambios económicos alrededor del mundo (excepto Estados Unidos), principalmente a la subida de precios de las acciones que consideran infravaloradas en los mercados en los que invierten. Además, para acotar sus posiciones utilizan índices derivados. Desafortunadamente no se localizaron fondos liquidados que hayan seguido esta estrategia por un periodo mínimo de 45 meses. Sin embargo, se cuenta con los resultados de los hedge funds activos. En ellos, la regresión con el índice $1^{47}$ arrojó coeficientes negativos y estadísticamente significativos para las sorpresas default premium y term structure, lo que indica que cuando estos fondos realizan inversiones en renta fija prefieren hacerlo a corto plazo, de modo que cuando los tipos de interés de los bonos de gobierno a corto plazo aumentan, la rentabilidad de estos hedge funds también. Por otro lado, el coeficiente de la sorpresa residual del mercado resultó positivo y estadísticamente significativo, algo que era de esperarse debido a que estos gestores se centran en el análisis de los activos que consideran infravalorados por los mercados internacionales, de manera que un aumento en las cotizaciones les generará altas rentabilidades para este tipo de fondos.

los cuatro grupos, respectivamente. Además, se observa que la regresión con el índice 1 para los hedge funds liquidados que aplican la global established tiene una bondad de ajuste de $67 \%$, mientras que para los activos es de $70 \%, 70 \%, 73 \%$ y $77 \%$, respectivamente.

${ }^{47}$ Para los hedge funds activos con estrategia global international, el índice de rentabilidad 1 agrupa $57 \%$ de los factores comunes que explican las interrelaciones entre los fondos que la adoptan. Además, la regresión del modelo con el índice 1 para estos hedge funds tiene una bondad de ajuste de $60 \%$. 
Global macro. Para el propósito de esta investigación, los hedge funds que adoptan la estrategia global macro son fundamentales, ya que se trata de fondos de gran tamaño que buscan oportunidades de inversión en los cambios de las variables macroeconómicas de las economías globales, típicamente variaciones en los tipos de interés y en los tipos de cambio, haciendo un fuerte uso del apalancamiento y de los productos derivados. Sus gestores buscan desequilibrios en el comportamiento de las variables macroeconómicas entre países, por lo que actúan en las economías de todo el mundo, principalmente en los mercados más líquidos, como el de deuda pública o el de divisas. Si el gestor considera que la política monetaria vigente de un país es inconsistente, apostará por una devaluación en dicho país, generalmente a través de ventas al descubierto, con la idea de recomprar la divisa a un precio menor (después de la devaluación) y devolverla a quien se la pidió prestada. De acuerdo con los resultados, en la regresión con el índice $1^{48}$ para los hedge funds liquidados, casi todas las sorpresas macroeconómicas son estadísticamente significativas (cuatro de cinco). ${ }^{49}$ El default premium y el crecimiento de la producción industrial obtuvieron coeficientes negativos, mientras que la sorpresa residual del mercado, uno positivo. Sin embargo, los resultados obtenidos para los hedge funds activos que siguen la global macro son menos alentadores, ya que tanto en la regresión con el índice 1, como en la regresión con el índice 2, sólo dos de las cinco sorpresas son estadísticamente significativas. Además, los signos de los coeficientes son contrarios en una y en otra regresión. Estos resultados tan poco claros pueden tener su origen en la enorme heterogeneidad de las subestrategias, ya que la cartera de estos fondos puede incluir acciones, bonos, divisas, materias primas y productos derivados, entre otros. ${ }^{50}$ Además, invierten en cualquier tipo de economía, tanto desarrolladas

${ }^{48}$ Para los hedge funds liquidados de la estrategia global macro, el índice de rentabilidad 1 agrupa $71 \%$ de los factores comunes que explican las interrelaciones entre los fondos que siguen esta estrategia. Y para los activos, el índice 1 agrupa $44 \%$ y el índice 2, 13\%. Además, se halló que la regresión con el índice 1 para los hedge funds liquidados tiene una bondad de ajuste de $74 \%$, mientras que para los activos es de $40 \%$, y de $47 \%$ al utilizar el índice 2 . La descripción de la regresión con el índice 2 se debe a que al usarlo se obtuvo una bondad de ajuste superior (47\%) a la obtenida con el índice $1(40 \%)$.

${ }^{49}$ La sorpresa term structure obtiene un signo negativo pero no es estadísticamente significativo.

${ }^{50}$ Brealey y Kaplanis (2001) se encuentran con el mismo problema y argumentan que mientras que para algunas estrategias existen factores de exposición que son obvios, para la global macro no son tan claros, ya que existe una considerable heterogeneidad entre los hedge funds que la siguen debido a la gran variedad de clase de activos en los que invierten. Por otro lado, mencionan que el conocimiento acerca del impacto de los hedge funds sobre un mercado en particular es limitado, sobre todo por esta misma naturaleza heterogénea. 
como emergentes. Los gestores de esta estrategia invierten en una amplia gama de productos realizando apuestas direccionales (alcistas o a la baja) y son guiados por sus expectativas de evolución de los mercados en relación a las variables macroeconómicas de los distintos países.

Long only/leverage. Los gestores de hedge funds que siguen la estrategia long only/leverage, como su nombre lo indica, son los que hacen mayor uso del apalancamiento con el objetivo de obtener mejores rentabilidades y ganar así la comisión variable. ${ }^{51}$ De acuerdo con ello, la sorpresa macroeconómica a la que deben poner especial atención es la term structure, ya que ésta comprende los tipos de interés de los bonos gubernamentales a corto y a largo plazo. Los resultados indican que en la regresión con el índice $1^{52}$ para los hedge funds liquidados se obtuvo un coeficiente positivo en la term structure, pero no es estadísticamente significativo. Contrario a esto, en los resultados obtenidos con el índice 1 para los fondos activos se observa que dicha sorpresa si es estadísticamente significativa, pero el coeficiente obtuvo un signo negativo. Por tanto, los resultados para esta estrategia no son claros, ya que, en principio, los hedge funds que la usan deberían tener una exposición positiva a la term structure,,$^{53}$ debido a que las estrategias que hacen uso de elevados niveles de apalancamiento regularmente implican invertir en la deuda de los gobiernos a largo plazo, de modo que al aumentar la rentabilidad de los bonos gubernamentales también aumentaría la rentabilidad de estos fondos.

Market neutral. Las subestrategias que forman parte de la estrategia market neutral se caracterizan por tratar de diversificar o neutralizar el riesgo de mercado. En principio, éste es generalmente reducido, pero requiere de un fuerte apalancamiento para obtener una rentabilidad satisfactoria. Entre las principales subestrategias dentro de la categoría market neutral se cuentan las siguientes: fixed inco-

${ }^{51}$ Utilizan la denominada high water mark, que es una meta de rentabilidad a la que el fondo pretende llegar o incluso rebasar. Esta meta es una garantía de que el gestor sólo cobra por el éxito efectivo (comisión variable).

${ }^{52}$ Para los hedge funds liquidados con estrategia long only/leverage, el índice 1 agrupa 55\% de los factores comunes que explican las interrelaciones entre los fondos que la siguen. Para los activos, el índice 1 también agrupa 55\%. Además, se halló que la regresión con el índice 1 para los liquidados tiene una bondad de ajuste de $55 \%$, mientras que para los activos es de $73 \%$.

${ }^{53}$ Una posible explicación se encuentra en el reducido número de hedge funds activos y liquidados que siguen esta estrategia, en la base de datos construida, de modo que los resultados en este caso pueden ser inestables. 
me arbitrage, fixed income convertible bonds, fixed income mortgage-backed, relative value, long-short. En teoría, se trata de procedimientos más conservadores en los que el gestor toma posiciones largas y cortas por la misma cuantía en valores de empresas. De igual manera, puede comprar bonos gubernamentales de un país y vender en corto bonos de otro con el fin de duplicar el valor de los bonos comprados en términos de tipo de interés y madurez. Por tanto, estos gestores intentan no seguir la tendencia del mercado y apuestan por los diferenciales entre dos clases de activos. Consideran que la exposición neta al riesgo de mercado disminuye si tiene igualdad en las posiciones largas y cortas en los mercados de renta variable y/o de renta fija. Dadas estas características, los hedge funds que usan la market neutral tendrán una importante relación con las sorpresas default premium y term structure. Los resultados muestran que en la regresión con el índice $1^{54}$ para los hedge funds liquidados se obtuvieron coeficientes negativos para el default premium y la term structure, pero sólo para la primera resultó estadísticamente significativo. De igual manera, en los resultados de la regresión con el índice 1 para los hedge funds activos, se obtuvieron coeficientes negativos para las sorpresas default premium y term structure, pero la significación estadística varía dependiendo de cada uno de los diez grupos analizados para esta estrategia (en siete de ellos, el default premium es estadísticamente significativo, pero en sólo dos la term structure lo es). Esto hace pensar que los fondos con estrategia market neutral están más orientados a la inversión a largo plazo en bonos de los gobiernos, ya que al disminuir la rentabilidad de éstos, aumenta el diferencial entre la deuda de las empresas y la del gobierno, provocando una disminución en la rentabilidad de estos hedge funds.

Sector. Los hedge funds que recurren a la estrategia sector invierten en compañías de diversos sectores de la economía. Las principales ramas en las que realizan operaciones de compra y venta de activos son: asistencia médica y biotecnología, servicios financieros, comidas y bebidas, comunicaciones y multimedia, recursos naturales, petróleo y gas, inmobiliaria, tecnología, transporte, energía,

\footnotetext{
${ }^{54}$ Para los hedge funds liquidados que siguen la market neutral, el índice de rentabilidad 1 agrupa $46 \%$ de los factores comunes que explican las interrelaciones entre ellos, mientras que para los activos, el mismo índice agrupa 60\%, 37\%, 41\%, 39\%, 48\%, 38\%, 46\%, 53\%, 46\% y 38\%, en cada uno de los diez grupos, respectivamente. Además, encontramos que la regresión con el índice 1 para los fondos liquidados que adoptan esta estrategia tiene una bondad de ajuste de $69 \%$, mientras que para los activos es de $39 \%, 28 \%, 84 \%, 41 \%, 50 \%, 57 \%, 56 \%, 56 \%, 63 \%$ y $55 \%$, respectivamente.
} 
metales y minería, miscelánea, entre otros. Los gestores de estos fondos buscan desequilibrios en los precios de los activos de estos sectores con el propósito de obtener beneficios. Por ello, la sorpresa macroeconómica a la que deben prestar especial atención estos hedge funds es el crecimiento de la producción industrial, ya que ésta representa los cambios más importantes dentro de las ramas en las que tienen sus inversiones. En los resultados se puede comprobar que en la regresión con el índice $1^{55}$ para los fondos activos y liquidados se obtuvieron coeficientes negativos y estadísticamente significativos en el caso del crecimiento de la producción industrial. ${ }^{56}$ Es decir, estos hedge funds obtienen rentabilidades positivas en presencia de una disminución de la actividad de la industria, lo que indicaría que apuestan más por una tendencia a la baja que por una alcista en las ramas en las que invierten. No obstante, este resultado es poco consistente, ya que en principio deberían verse beneficiados por el crecimiento en la actividad industrial.

Short-sellers. Los hedge funds que siguen esta estrategia van en contra del movimiento del mercado, tomando posiciones cortas o largas, según sus valoraciones. Esta opción se basa principalmente en las ventas al descubierto, es decir, el gestor pide prestado un activo que considera sobrevalorado para venderlo con la esperanza de que posteriormente disminuya su precio y comprarlo más barato para devolverlo a quien se lo pidió prestado. Dado que la definición de esta estrategia es muy precisa, la sorpresa macroeconómica más importante para estos hedge funds es la residual del mercado. Según los resultados, en la regresión con el índice $1^{57}$ para los fondos activos y liquidados se obtuvieron coeficientes negativos y estadísticamente significativos para la sorpresa residual del mercado, lo que indica que si la tendencia es a la baja, la rentabilidad de estos hedge funds au-

\footnotetext{
${ }^{55}$ Para los hedge funds liquidados con estrategia sector, el índice de rentabilidad 1 agrupa 50\% de los factores comunes que explican las interrelaciones entre ellos, mientras que para los activos, el mismo índice agrupa $40 \%$ y 50\%, en cada uno de los dos grupos, respectivamente. Además, se encuentra que la regresión con el índice 1 para los fondos liquidados tiene una bondad de ajuste de $61 \%$, mientras que para los activos es de $63 \%$ y $50 \%$, respectivamente.

${ }^{56}$ Excepto en la regresión con el índice 1 para el segundo grupo de los hedge funds activos con estrategia sector, ya que -aunque el crecimiento de la producción industrial tiene signo negativono es estadísticamente significativo.

${ }^{57}$ Para los hedge funds liquidados que adoptaron la estrategia short-sellers, el índice de rentabilidad 1 agrupa $71 \%$ de los factores comunes que explican las interrelaciones entre ellos, mientras que para los activos, el mismo índice agrupa 63\%. Además, se halló que la regresión con el índice 1 para los fondos liquidados tiene una bondad de ajuste de $47 \%$, mientras que para los activos es de $67 \%$.
} 
mentará y viceversa. Así, se trata de un tipo de fondo contrario a los movimientos del mercado y que en situaciones de crisis suele tener buena rentabilidad.

Inflación no esperada y residual del mercado. Tras analizar las relaciones encontradas entre los hedge funds (activos y liquidados) y las sorpresas default premium, term structure y crecimiento de la producción industrial, es el turno de describir las relaciones encontradas con las sorpresas de inflación no esperada y residual del mercado. Para los fondos liquidados de casi todas las estrategias, el coeficiente de la inflación no esperada obtuvo un signo negativo, lo que indica que las sorpresas en la inflación afectaron negativamente su rentabilidad. Respecto a los fondos activos, los coeficientes de la inflación no esperada no están tan definidos, ya que con las estrategias event driven, global established, sector y market neutral se obtuvieron signos negativos, mientras que con las estrategias global emerging, global internacional, global macro, long only/leverage y shortsellers fueron positivos. Sin embargo, en la mayoría de las regresiones de los fondos tanto activos como liquidados, esta sorpresa resultó no ser estadísticamente significativa. El coeficiente negativo de la inflación no esperada sólo fue significativo para los hedge funds liquidados que adoptaron la global macro. ${ }^{58}$ Por otra parte, el coeficiente de la sorpresa residual del mercado obtuvo un signo positivo en casi todas las estrategias de los fondos tanto activos como liquidados, lo que significa que a la mayoría les afecta directamente la tendencia del mercado, independientemente de la estrategia que sigan, incluida la market neutral. La excepción es la estrategia short-sellers, que va en contra del movimiento del mercado, y esto se ve reflejado en el signo negativo y estadísticamente significativo del coeficiente de la sorpresa residual del mercado, tanto en las regresiones de los fondos activos como en las de los liquidados. Finalmente, los coeficientes obtenidos para la sorpresa en el crecimiento de la producción industrial fueron estadísticamente significativos en casi todas las estrategias seguidas por los hedge funds liquidados ${ }^{59} \mathrm{y}$ activos ${ }^{60}$, lo que indica que la actividad económica también les afecta, independientemente de su estrategia.

\footnotetext{
${ }^{58}$ En la estrategia short-sellers de los hedge funds liquidados esta sorpresa también resultó estadísticamente significativa aunque con signo positivo.

${ }^{59}$ Excepto para la estrategia long only/leverage, en la que únicamente la sorpresa default premium resultó estadísticamente significativa debido a la preferencia de estos fondos por la inversión a largo plazo.

${ }^{60}$ Excepto en la mayor parte de los grupos de los hedge funds que siguen la estrategia market neutral, ya que, al parecer, la actividad económica no tiene una influencia representativa sobre ellos.
} 


\section{CONCLUSIONES}

Con el propósito de analizar la influencia que tienen los factores de riesgo macroeconómicos sobre la rentabilidad de los hedge funds activos y liquidados por estrategia seguida, se ha estimado un modelo multifactor macroeconómico que consiguió reflejar las relaciones existentes entre los índices de rentabilidad (obtenidos mediante análisis factoriales aplicados a cada una de las estrategias seguidas por los fondos que conforman una base de datos construida manualmente con la información publicada en la revista MARHedge Performance \& Evaluation Directory, de 1999 a 2006) y diversas sorpresas macroeconómicas (elaboradas a partir de un modelo autorregresivo, primeras diferencias y cálculos aritméticos). Después de construir las variables dependientes e independientes, el modelo se estimó en series temporales mediante MCO y la matriz de White.

La base de datos quedó conformada por 3097 hedge funds activos y 1 761 liquidados. Después de seleccionar aquellos de los que se disponía información sobre sus rentabilidades, la muestra disminuyó a 2645 activos y 1016 liquidados. Sin embargo, tras una segunda selección que descartó a los fondos que carecían de información sobre su rentabilidad mensual, la muestra final quedó formada por 994 hedge funds activos y 190 liquidados, debido a que la mayoría de los fondos reportan su rentabilidad de manera anual. Para cada uno de los fondos activos se dispuso de 54 rentabilidades mensuales, mientras que para cada uno de los liquidados se dispuso de 45. La restricción del periodo de análisis se impuso con el objetivo de abarcar el lapso más amplio posible.

Mediante la aplicación del análisis factorial a cada uno de los grupos de hedge funds (activos y liquidados) por estrategia seguida, se obtuvo el número mínimo de dimensiones capaces de explicar el máximo de información contenida en las rentabilidades mensuales. A partir de los factores extraídos mediante el método de ejes principales, se calcularon las puntuaciones factoriales para cada observación mensual, las cuales fueron empleadas como índices de rentabilidad por estrategia seguida y se utilizaron como variables dependientes en el modelo multifactor, mientras que como variables independientes se utilizaron las sorpresas macroeconómicas construidas. La inflación no esperada se obtuvo a partir un modelo autorregresivo; el default premium y la term structure, a partir de cálculos aritméticos, y la sorpresa en el crecimiento de la producción industrial se calculó mediante primeras diferencias, mientras la residual del mercado es el residuo del modelo de regresión lineal que utiliza como variables explicativas al resto de las sorpresas macroeconómicas. 
Una vez estimado el modelo multifactor para cada uno de los índices de rentabilidad de los hedge funds activos y liquidados por estrategia seguida, éste fue sometido a los contrastes de especificación y diagnóstico (estacionariedad, multicolinealidad, autocorrelación, normalidad, heterocedasticidad y linealidad) requeridos para comprobar su solidez y calidad. Después de pasar las pruebas básicas, se procedió a la interpretación de los resultados de las regresiones de tipo ARMA por estrategia seguida. A partir de los resultados se puede concluir que las estrategias se agrupan en tres grandes familias: las neutrales al riesgo de mercado; las direccionales, que apuestan por un determinado comportamiento del mercado, y las oportunistas, que buscan situaciones especiales en relación a eventos corporativos.

Para los hedge funds liquidados de casi todas las estrategias, el coeficiente de la inflación no esperada obtuvo un signo negativo, lo que indica que las sorpresas en la inflación afectaron negativamente su rentabilidad. En el caso de los hedge funds activos, los coeficientes de esta sorpresa no estuvieron tan definidos. Además, en la mayoría de las regresiones, resultó no ser estadísticamente significativa, excepto para los fondos liquidados con estrategia global macro. Por otra parte, el coeficiente de la residual del mercado obtuvo un signo positivo en casi todas las estrategias de los hedge funds, tanto activos como liquidados, lo que significa que a la mayoría de los fondos les afecta directamente la tendencia del mercado, independientemente de la estrategia que sigan. La excepción fueron los hedge funds con estrategia short-sellers, que obtuvieron un signo negativo y estadísticamente significativo. Conviene destacar que los coeficientes obtenidos para el crecimiento de la producción industrial fueron estadísticamente significativos en casi todas las estrategias, lo que indica que la actividad económica afecta a los hedge funds independientemente de la estrategia que adopten. Por último, las sorpresas default premium y term structure fueron analizadas en cada una de las estrategias debido a su gran relevancia en este sector, aunque en la mayor parte de los casos se encontraron las relaciones esperadas. En general, la mayoría de las exposiciones de los índices de rentabilidad a las sorpresas macroeconómicas se explican en términos de la filosofía de inversión de los hedge funds.

\section{REFERENCIAS BIBLIOGRÁFICAS}

Antoniou, Antonios; Garrett, Ian, y Priestley, Richard. (1998), "Macroeconomic Variables as common pervasive risk factors and the empirical content of the Arbitrage Pricing Theory”, Journal of Empirical Finance, 5 (3), pp. 221-240. 
Azeez, A. A. y Yonezawa, Yazuhiro (2006), "Macroeconomic factors and the empirical content of the Arbitrage Pricing Theory in the Japanese Stock Market", Japan and the World Economy, 18 (4), pp. 568-591.

Bailey, Warren, y Chan, K. C. (1993), "Macroeconomic Influences and the Variability of the Commodity Futures Basis", The Journal of Finance, 48 (2), pp. 555-573.

Berry, Michel A.; Burmeister, Edwin, y McElroy, Marjorie B. (1988), "Sorting Out Risks Using Known APT Factors”, Financial Analysts Journal, 44 (2), pp. 29-42.

Brealey, Richard. A., y Kaplanis, Evi (2001), "Changes in the Factor Exposures of Hedge Funds", working paper, Banco de Inglaterra, enero.

Brown, Stephen J.; Goetzmann, William N., y Ibbotson, Roger (1999), "Offshore Hedge Funds: Survival and Performance 1989-1995”, Journal of Business, 72 (1), pp. 91-118.

Burmeister, Edwin; Roll, Richard, y Ross, Stephen A. (2003), "Using Macroeconomic Factors to Control Portfolio Risk", working paper, BIRR Portfolio Analysis, Inc., marzo.

Caslin, John (2004), Hedge Funds, Glasow, Bell \& Bain.

Chan, K. C.; Chen, Nai-Fu, y Hsieh, David (1985), "An exploratory investigation of firm size effect”, Journal of Financial Economics, 14 (3), pp. 451-471.

Chen, Nai-Fu; Roll, Richard, y Ross, Stephen (1986), "Economic Forces and the Stock Market”, Journal of Business, 59 (3), pp. 383-403.

Chen, Su-Jane, y Jordan, Bradford D. (1993), "Some empirical tests in the Arbitrage Pricing Theory: Macrovariables vs. Derived Factors", Journal of Banking and Finance, 17 (1), pp. 65-89.

Clare, Andrew D. y Thomas, Stephen H. (1994), "Macroeconomic Factors, the APT and the UK Stockmarket", Journal of Business Finance \& Accounting, 21 (3), pp. 309-330.

Connor, Gregory (1995), "The Three Types of Factor Models: A Comparison of Their Explanatory Power", Financial Analysts Journal, 51 (3), pp. 42-46.

Das, Nandita; Kish, Richard J., y Muething, David L. (2005), "Modeling Hedge Fund Returns", Financial Decisions, 17(3), pp. 1-23 (article 4).

Erdinç, Altay (2003), "The Effect of Macroeconomic Factors on Asset Returns: A comparative Analysis of the German and the Turkish Stock Markets in an APT Framework", working paper 48, Martin-Luther-Universität Halle-Wittenberg, mayo.

Gómez-Bezares, Fernando; Madariaga, José Antonio, y Santibáñez, Javier (1994), Valoración de acciones en la bolsa española, Bilbao, Desclee De Brouwer.

Li, Lingfeng (2002), "Macroeconomic Factors and the Correlation of Stock and Bond 
44 ECONOMÍA: TEORÍA Y PRÁCTICA • Nueva Época, número 42, enero-junio 2015

Returns", working paper 02-46, Yale International Center for Finance, Yale University.

Liang, Bing (2001), “Hedge Fund Performance: 1990-1999”, Financial Analysts Journal, 57 (1), pp.11-18

López-Pascual, Joaquín, y Camarero, Santiago (2013), “Analysing hedge fund strategies through the use of an option based approach", Revista Española de Financiación y Contabilidad, 42 (158), pp. 167-186.

MARHedge Performance \& Evaluation Directory (1999 - 2006), primer y segundo semestres de 1999, 2000, 2001, 2003 y 2004; primer, segundo, tercero y cuarto trimestres de 2005; primer y segundo trimestres de 2006. 